\title{
Second order superintegrable systems in conformally flat spaces. III. Three-dimensional classical structure theory
}

\author{
E. G. Kalnins \\ Department of Mathematics and Statistics, University of Waikato, Hamilton, \\ New Zealand \\ J. M. Kress \\ School of Mathematics, The University of New South Wales, \\ Sydney NSW 2052, Australia \\ W. Miller, Jr. \\ School of Mathematics, University of Minnesota, Minneapolis, Minnesota 55455
}

(Received 8 July 2005; accepted 22 July 2005; published online 11 October 2005)

\begin{abstract}
This paper is part of a series that lays the groundwork for a structure and classification theory of second-order superintegrable systems, both classical and quantum, in real or complex conformally flat spaces. Here we consider classical superintegrable systems with nondegenerate potentials in three dimensions. We show that there exists a standard structure for such systems, based on the algebra of $3 \times 3$ symmetric matrices, and that the quadratic algebra always closes at order 6 . We show that the spaces of truly second-, third-, fourth-, and sixth-order constants of the motion are of dimension $6,4,21$, and 56, respectively, and we construct explicit bases for the fourth- and sixth order constants in terms of products of the second order constants. () 2005 American Institute of Physics. [DOI: 10.1063/1.2037567]
\end{abstract}

\section{INTRODUCTION AND EXAMPLES}

The goal of this series of papers, ${ }^{1,2}$ is a structure and classification theory of second-order superintegrable systems, both classical and quantum, in conformally flat spaces. An $n$-dimensional Riemannian space is conformally flat if and only if it admits a set of local coordinates $x_{1}, \ldots, x_{n}$ such that the contravariant metric tensor takes the form $g^{i j}=\delta^{i j} / \lambda(\mathbf{x})$. In other words, the metric is $\mathrm{d} s^{2}=\lambda(\mathbf{x})\left(\sum_{i=1}^{n} \mathrm{~d} x_{i}^{2}\right)$. A classical superintegrable system $\mathcal{H}=\Sigma_{i j} g^{i j} p_{i} p_{j}+V(\mathbf{x})$ on the phase space of this manifold is one that admits $2 n-1$ functionally independent generalized symmetries (or constants of the motion) $\mathcal{S}_{k}, k=1, \ldots, 2 n-1$ with $\mathcal{S}_{1}=\mathcal{H}$. That is, $\left\{\mathcal{H}, \mathcal{S}_{k}\right\}=0$ where

$$
\{f, g\}=\sum_{j=1}^{n}\left(\partial_{x_{j}} f \partial_{p_{j}} g-\partial_{p_{j}} f \partial_{x_{j}} g\right)
$$

is the Poisson bracket for functions $f(\mathbf{x}, \mathbf{p}), g(\mathbf{x}, \mathbf{p})$ on phase space. ${ }^{3-10}$ Note that $2 n-1$ is the maximum possible number of functionally independent symmetries and, locally, such symmetries always exist. (In this paper $n=3$ so we have five functionally independent symmetries.) The main interest is in symmetries that are polynomials in the $p_{k}$ and are globally defined, except for lower dimensional singularities such as poles and branch points. Many tools in the theory of Hamiltonian systems have been brought to bear on superintegrable perintegrable systems, such as $R$-matrix theory, Lax pairs, exact solvability, quasiexact solvability, and the Jacobi metric. ${ }^{11-15}$ However, the most detailed and complete results are obtained from separation of variables methods in those cases where they are applicable. Standard orthogonal separation of variables techniques are associated with second-order symmetries, e.g., Refs. 16-21 and multiseparable Hamiltonian systems provide numerous examples of superintegrability. In these papers we concentrate on second-order 
superintegrable systems, that is those in which the symmetries take the form $\mathcal{S}=\sum a^{i j}(\mathbf{x}) p_{i} p_{j}$ $+W(\mathbf{x})$, quadratic in the momenta.

There is an analogous definition for second-order quantum superintegrable systems with Schrödinger operator

$$
H=\Delta+V(\mathbf{x}), \quad \Delta=\frac{1}{\sqrt{g}} \sum_{i j} \partial_{x_{i}}\left(\sqrt{g} g{ }^{i j}\right) \partial_{x_{j}},
$$

the Laplace-Beltrami operator plus a potential function. ${ }^{16}$ Here there are $2 n-1$ second-order symmetry operators

$$
S_{k}=\frac{1}{\sqrt{g}} \sum_{i j} \partial_{x_{i}}\left(\sqrt{g} a_{(k)}^{i j}\right) \partial_{x_{j}}+W^{(k)}(\mathbf{x}), \quad k=1, \ldots, 2 n-1
$$

with $S_{1}=H$ and $\left[H, S_{k}\right] \equiv H S_{k}-S_{k} H=0$. Again multiseparable systems yield many examples of superintegrability, though not all multiseparable systems are superintegrable and not all secondorder superintegrable systems are multiseparable. There is also a quantization problem in extending the results for classical systems to operator systems. This problem turns out to be not difficult to solve for the nondegenerate systems that we study in this paper.

Superintegrable systems can (1) be solved explicitly, and (2) they can be solved in multiple ways. It is the information gleaned from comparing the distinct solutions and expressing one solution set in terms of another that is a primary reason for their interest.

We give a few simple three-dimensional (3D) examples to illustrate some of the main features of superintegrable systems. (To make clearer the connection with quantum theory and Hilbert space methods we shall, for these examples alone, adopt standard physical normalizations, such as using the factor $-\frac{1}{2}$ in front of the free Hamiltonian.) Consider the Schrödinger eigenvalue equation $H \Psi=E \Psi$ or $\left(\hbar=m=1, x_{1}=x, x_{2}=y, x_{3}=z\right)$

$$
H \Psi=-\frac{1}{2}\left(\frac{\partial^{2}}{\partial x^{2}}+\frac{\partial^{2}}{\partial y^{2}}+\frac{\partial^{2}}{\partial z^{2}}\right) \Psi+V(x, y, z) \Psi=E \Psi .
$$

The generalized anisotropic oscillator corresponds to the four-parameter potential

$$
V(x, y, z)=\frac{\omega^{2}}{2}\left(x^{2}+y^{2}+4(z+\rho)^{2}\right)+\frac{1}{2}\left[\frac{k_{1}^{2}-\frac{1}{4}}{x^{2}}+\frac{k_{2}^{2}-\frac{1}{4}}{y^{2}}\right] .
$$

(This potential is "nondegenerate" in the precise sense that we will explain in Sec. III.) The corresponding Schrödinger equation has separable solutions in five coordinate systems, Cartesian coordinates, cylindrical polar coordinates, cylindrical elliptic coordinates, cylindrical parabolic coordinates, and parabolic coordinates. The energy eigenstates for this equation are degenerate and important special function identities arise by expanding one basis of separable eigenfunctions in terms of another. A second order symmetry operator for this equation is a second-order linear differential operator $S$ such that $[H, S]=0$ where $[A, B]=A B-B A$. A basis for these operators is

$$
\begin{gathered}
M_{1}=\partial_{x}^{2}-\omega^{2} x^{2}+\frac{k_{1}^{2}-\frac{1}{4}}{x^{2}}, \quad M_{2}=\partial_{y}^{2}-\omega^{2} y^{2}-\frac{k_{2}^{2}-\frac{1}{4}}{y^{2}}, \\
P=\partial_{z}^{2}-4 \omega^{2}(z+\rho)^{2}, \quad L=L_{12}^{2}-\left(k_{1}^{2}-\frac{1}{4}\right) \frac{y^{2}}{x^{2}}-\left(k_{2}^{2}-\frac{1}{4}\right) \frac{x^{2}}{y^{2}}-\frac{1}{2}, \\
S_{1}=-\frac{1}{2}\left(\partial_{x} L_{13}+L_{13} \partial_{x}\right)+\rho \partial_{x}^{2}+(z+\rho)\left(\omega^{2} x^{2}-\frac{k_{1}^{1}-\frac{1}{4}}{x^{2}}\right),
\end{gathered}
$$




$$
S_{2}=-\frac{1}{2}\left(\partial_{y} L_{23}+L_{23} \partial_{y}\right)+\rho \partial_{y}^{2}+(z+\rho)\left(\omega^{2} y^{2}-\frac{k_{2}^{2}-\frac{1}{4}}{y^{2}}\right),
$$

where $L_{i j}=x_{i} \partial_{x_{j}}-x_{j} \partial_{x_{i}}$. Remarkably, these symmetries generate a "quadratic algebra" that closes at level six. Indeed, the nonzero commutators of the above basis are

$$
\left[M_{1}, L\right]=\left[L, M_{2}\right]=Q, \quad\left[L, S_{1}\right]=\left[S_{2}, L\right]=B, \quad\left[M_{i}, S_{i}\right]=A_{i}, \quad\left[P, S_{i}\right]=-A_{i} .
$$

Nonzero commutators of the basis symmetries with $Q$ (fourth-order symmetries) are expressible in terms of the second-order symmetries,

$$
\begin{gathered}
{\left[M_{i}, Q\right]=\left[Q, M_{2}\right]=4\left\{M_{1}, M_{2}\right\}+16 \omega^{2} L, \quad\left[S_{1}, Q\right]=\left[Q, S_{2}\right]=4\left\{M_{1}, M_{2}\right\},} \\
{[L, Q]=4\left\{M_{1}, L\right\}-4\left\{M_{2}, L\right\}+16\left(1-k_{1}^{2}\right) M_{1}-16\left(1-k_{2}^{2}\right) M_{2} .}
\end{gathered}
$$

There are similar expressions for commutators with $B$ and the $A_{i}$. Also the squares of $Q, B, A_{i}$ and products such as $\{Q, B\}$ (all sixth-order symmetries) are expressible in terms of second-order symmetries. For example,

$$
\begin{aligned}
Q^{2}= & \frac{8}{3}\left\{L, M_{1}, M_{2}\right\}+8 \omega^{2}\{L, L\}-16\left(1-k_{1}^{2}\right) M_{1}^{2}-16\left(1-k_{2}^{2}\right) M_{2}^{2}+\frac{64}{3}\left\{M_{1}, M_{2}\right\} \\
& -\frac{128}{3} \omega^{2} L-128 \omega^{2}\left(1-k_{1}^{2}\right)\left(1-k_{2}^{2}\right), \\
\{Q, B\}= & -\frac{8}{3}\left\{M_{2}, L, S_{1}\right\}-\frac{8}{3}\left\{M_{1}, L, S_{2}\right\}+16\left(1-k_{1}^{2}\right)\left\{M_{2}, S_{2}\right\}+16\left(1-k_{2}^{2}\right)\left\{M_{1}, S_{1}\right\} \\
& -\frac{64}{3}\left\{M_{1}, S_{2}\right\}-\frac{64}{3}\left\{M_{2}, S_{1}\right\} .
\end{aligned}
$$

Here $\left\{C_{1}, \ldots, C_{j}\right\}$ is the completely symmetrized product of operators $C_{1}, \ldots, C_{j}$. (For complete details of all the possible products and commutators, see Ref. 22.) The point is that the algebra generated by products and commutators of the second order symmetries closes at order 6 .

Another example in Euclidean space is given by the Schrödinger equation with threeparameter extended Kepler-Coulomb potential,

$$
\left(\frac{\partial^{2} \Psi}{\partial x^{2}}+\frac{\partial^{2} \Psi}{\partial y^{2}}+\frac{\partial^{2} \Psi}{\partial z^{2}}\right)+\left[2 E+\frac{2 \alpha}{\sqrt{x^{2}+y^{2}+z^{2}}}-\left(\frac{k_{1}^{2}-\frac{1}{4}}{x^{2}}+\frac{k_{2}^{2}-\frac{1}{4}}{y^{2}}\right)\right] \Psi=0 .
$$

This equation admits separable solutions in the four coordinates systems: spherical, sphero conical, prolate spheroidal, and parabolic coordinates. Again the bound states are degenerate and important special function identities arise by expanding one basis of separable eigenfunctions in terms of another. However, the space of second-order symmetries is only five dimensional and, although there are useful identities among the generators and commutators that enable one to derive spectral properties algebraically, there is no finite quadratic algebra structure. The key difference with our first example is, as we shall show later, that the three-parameter KeplerCoulomb potential is degenerate and it cannot be extended to a four-parameter potential.

An example on the three-sphere is given by

$$
g^{i j}=\delta_{i j} z_{i}-z_{i} z_{j}, \quad 1 \leqslant i, j \leqslant 3 .
$$

Then $\operatorname{det}\left(g^{i j}\right)=g^{-1}=z_{1} z_{2} z_{3}(1-z)$ where $z=z_{1}+z_{2}+z_{3}$ and

$$
g_{i j}=\frac{1}{1-z}+\frac{\delta_{i j}}{z_{i}} .
$$

Thus $\mathrm{d} s^{2}=\sum_{i, j=1}^{3} g_{i j} \mathrm{~d} z_{i} \mathrm{~d} z_{j}$. To identify this space we introduce Cartesian coordinates $x_{0}, x_{1}, x_{2}, x_{3}$ in four-dimensional Euclidean space and restrict these coordinates by the conditions 


$$
x_{0}^{2}=1-z, \quad x_{1}^{2}=z_{1}, \quad x_{2}^{2}=z_{2}, \quad x_{3}^{2}=z_{3} .
$$

Note that $x_{0}^{2}+x_{1}^{2}+x_{2}^{2}+x_{3}^{2}=1$. Defining a metric $\mathrm{d} s^{2}$ by $\mathrm{d} s^{2}=\Sigma_{m=0}^{3}\left(\mathrm{~d} x_{m}\right)^{2}$ we find

$$
\mathrm{d} s^{2}=\frac{1}{4} \sum_{i, j=1}^{3}\left(\frac{1}{1-z}+\frac{\delta_{i j}}{z_{i}}\right) \mathrm{d} z_{i} \mathrm{~d} z_{j} .
$$

Thus the space corresponds to a portion of the three-sphere $S^{n}$. The Schrödinger equation is

$$
\left(\Delta+\sum_{i=1}^{3} \frac{\gamma_{i}}{z_{i}}+\frac{\gamma_{4}}{1-z}\right) \Psi=E \Psi
$$

where $\Delta$ is the Laplace-Beltrami operator. This is a nondegenerate potential. The six second-order operators

$$
\begin{gathered}
S_{i j}=4 z_{i} z_{j}\left(\partial_{z_{i}}-\partial_{z_{j}}\right)^{2}+4\left(g_{i} z_{j}-g_{j} z_{i}\right)\left(\partial_{z_{i}}-\partial_{z_{j}}\right)=S_{j i}, \quad 1 \leqslant i<j \leqslant 3, \\
S_{0 i}=4 z_{i}(1-z) \partial_{z_{i}}^{2}+4\left[g_{i}(1-z)-g_{0} z_{i}\right] \partial_{z_{i}}=S_{i 0}, \quad 1 \leqslant i \leqslant 3,
\end{gathered}
$$

for $g_{i}=1+\frac{1}{2} \sqrt{1-16 \gamma_{i}}$ form a basis for the space of second-order symmetries. In particular

$$
8 H=\sum_{i, j=1}^{n} S_{i j}+2 \sum_{i=1}^{n} S_{0 i} .
$$

This equation separates in six coordinate systems on the three sphere. Further it can be shown that the quadratic algebra generated by the second-order symmetries closes at order 6 .

For our last example we take the space with metric

$$
\mathrm{d} s^{2}=\lambda(A, B, C, D, E, \mathbf{x})\left(\mathrm{d} x^{2}+\mathrm{d} y^{2}+\mathrm{d} z^{2}\right),
$$

where

$$
\begin{aligned}
\lambda= & A(x+i y)+B\left(\frac{3}{4}(x+i y)^{2}+\frac{z}{4}\right)+C\left((x+i y)^{3}+\frac{1}{16}(x-i y)+\frac{3 z}{4}(x+i y)\right) \\
& +D\left(\frac{5}{16}(x+i y)^{4}+\frac{z^{2}}{16}+\frac{1}{16}\left(x^{2}+y^{2}\right)+\frac{3 z}{8}(x+i y)^{2}\right)+E,
\end{aligned}
$$

the nondegenerate potential is $V=\lambda(\alpha, \beta, \gamma, \delta, \epsilon, \mathbf{x}) / \lambda(A, B, C, D, E, \mathbf{x})$. If $A=B=C=D=0$ this is a superintegrable system on complex Euclidean space. The quadratic algebra always closes, and for general values of $A, B, C, D, E$ the space is not of constant curvature. This is an example of a superintegrable system that is Stäckel equivalent to a system on complex Euclidean space. We will take up the study of such systems in the next paper in this series.

Observed common features of superintegrable systems (and features that we make precise and verify in these papers) are that they are usually multiseparable and that the eigenfunctions of one separable system can be expanded in terms of the eigenfunctions of another. This is the source of nontrivial special function expansion theorems. ${ }^{23}$ The symmetry operators are in formal selfadjoint form and suitable for spectral analysis. Also, the quadratic algebra identities allow us to relate eigenbases and eigenvalues of one symmetry operator to those of another. Indeed the representation theory of the abstract quadratic algebra can be used to derive spectral properties of the second-order generators in a manner analogous to the use of Lie algebra representation theory to derive spectral properties of quantum systems that admit Lie symmetry algebras. ${ }^{23-26}$ (Note however that for superintegrable systems with nondegenerate potential, there is no first-order Lie symmetry.) 
Another common feature of quantum superintegrable systems is that they can be modified by a gauge transformation so that the Schrödinger and symmetry operators are acting on a space of polynomials. ${ }^{27}$ This is closely related to the theory of exactly and quasi exactly solvable systems. ${ }^{13,28}$ The characterization of ODE quasiexactly solvable systems as embedded in PDE superintegrable systems provides considerable insight into the nature of these phenomena.

The classical analogs of the above examples are obtained by the replacements $\partial_{x_{i}} \rightarrow p_{x_{i}}$. Commutators go over to Poisson brackets. The operator symmetries become second-order constants of the motion. Symmetrized operators become products of functions. The quadratic algebra relations simplify, the highest order terms agree with the operator case but there are fewer nonzero lower order terms.

Many examples of 3D superintegrable systems are known, although they have not been classified. ${ }^{29-34}$ Here, rather than focus on particular spaces and systems, we employ a theoretical method based on integrability conditions to derive structure common to all such systems, with a view to complete classification, at least for nondegenerate potentials. In this paper we consider classical superintegrable systems on a general 3D conformally flat spaces, real or complex, and uncover their common structure. We show that for systems with nondegenerate potentials there exists a standard structure based on the algebra of $3 \times 3$ symmetric matrices, and that the quadratic algebra closes at level 6. For two dimensional (2D) nondegenerate superintegrable systems we can show that the three functionally independent constants of the motion are (with one exception) also linearly independent, so at each regular point we can find a unique constant of the motion that matches a quadratic expression in the momenta at that point. However, for 3D systems we have only five functionally independent constants of the motion and the quadratic forms span a sixdimensional space. This is a major problem. However, for nondegenerate potentials we can prove the " $5 \Rightarrow 6$ Theorem" to show that the space of second-order constants of the motion is in fact six dimensional, there is a symmetry that is functionally dependent on the symmetries that arise from superintegrability, but linearly independent of them. With that result established, the treatment of the $3 \mathrm{D}$ case proceeds in analogy with the nondegenerate $2 \mathrm{D}$ case treated in Ref. 1 . Though the details are quite complicated, we show that the spaces of truly second-, third-, fourth-, and sixthorder constants of the motion are of dimension 6, 4, 21, and 56, respectively. Finally we construct explicit bases for the fourth- and sixth-order constants in terms of products of the second-order constants. These bases are our principal result. They guarantee closure of the quadratic algebra and provide a means for analyzing its structure. This paper is a major advance toward one of our goals, to obtain a demonstrably complete list of 3D superintegrable potentials.

In the next paper in this series we will show that all 3D superintegrable systems with nondegenerate potential are multiseparable. We will study the Stäckel transform, or coupling constant metamorphosis, ${ }^{35,36}$ for 3D classical superintegrable systems. This is a conformal transformation of a superintegrable system on one space to a superintegrable system on another space. We will prove that all nondegenerate 3D superintegrable systems are Stäckel transforms of constant curvature systems. We will also extend our results to the quantum analogs of $2 \mathrm{D}$ and $3 \mathrm{D}$ classical systems.

\section{CONFORMALLY FLAT SPACES IN THREE DIMENSIONS}

We assume that there is a coordinate system $x, y, z$ and a nonzero function $\lambda(x, y, z)$ $=\exp [G(x, y, z)]$ such that the Hamiltonian is

$$
\mathcal{H}=\frac{p_{1}^{2}+p_{2}^{2}+p_{3}^{2}}{\lambda}+V(x, y, z) .
$$

A quadratic constant of the motion (or generalized symmetry) 


$$
\mathcal{S}=\sum_{k, j=1}^{3} a^{k j}(x, y, z) p_{k} p_{j}+W(x, y, z) \equiv \mathcal{L}+W, \quad a^{j k}=a^{k j}
$$

must satisfy $\{\mathcal{H}, \mathcal{S}\}=0$. The conditions are thus

$$
\begin{gathered}
a_{i}^{i i}=-G_{1} a^{1 i}-G_{2} a^{2 i}-G_{3} a^{3 i}, \\
2 a_{i}^{i j}+a_{j}^{i i}=-G_{1} a^{1 j}-G_{2} a^{2 j}-G_{3} a^{3 j}, \quad i \neq j, \\
a_{k}^{i j}+a_{j}^{k i}+a_{i}^{j k}=0, \quad i, j, k \text { distinct }
\end{gathered}
$$

and

$$
W_{k}=\lambda \sum_{s=1}^{3} a^{s k} V_{s}, \quad k=1,2,3 .
$$

(Here a subscript $j$ denotes differentiation with respect to $x_{j}$.) The requirement that $\partial_{x_{\ell}} W_{j}$ $=\partial_{x_{j}} W_{\ell}, \ell \neq j$ leads from (9) to the second-order Bertrand-Darboux partial differential equations for the potential,

$$
\sum_{s=1}^{3}\left[V_{s j} \lambda a^{s \ell}-V_{s \ell} \lambda a^{s j}+V_{s}\left(\left(\lambda a^{s \ell}\right)_{j}-\left(\lambda a^{s j}\right)_{\ell}\right)\right]=0
$$

For second-order superintegrabilty in 3D there must be five functionally independent constants of the motion (including the Hamiltonian itself). Thus the Hamilton-Jacobi equation admits four additional constants of the motion

$$
\mathcal{S}_{h}=\sum_{j, k=1}^{3} a_{(h)}^{j k} p_{k} p_{j}+W_{(h)}=\mathcal{L}_{h}+W_{(h)}, \quad h=1, \ldots, 4 .
$$

We assume that the four functions $\mathcal{S}_{h}$ together with $\mathcal{H}$ are functionally independent in the sixdimensional phase space, i.e., that the differentials $\mathrm{d} \mathcal{S}_{h}, \mathrm{~d} \mathcal{H}$ are linearly independent. (Here the possible $V$ will always be assumed to form a vector space and we require functional independence for each such $V$ and the associated $W^{(h)}$. This means that we also require that the five quadratic forms $\mathcal{L}_{h}, \mathcal{H}_{0}$ are functionally independent.) We say that the functions are weakly functionally independent if $\mathrm{d} \mathcal{S}_{h}, \mathrm{~d} \mathcal{H}$ are linearly independent for nonzero potentials, but not necessarily for the zero potential.

\section{FUNCTIONAL LINEAR INDEPENDENCE}

We first shed some light on the relationship between functional independence and functional linear independence for the set $\left\{\mathcal{H}, \mathcal{S}_{1}, \ldots, \mathcal{S}_{4}\right\}$

Theorem 1: The functionally independent $\operatorname{set}\left\{\mathcal{H}, \mathcal{S}_{1}, \ldots, \mathcal{S}_{4}\right\}$ is also functionally linearly independent in the sense that if the relation $\Sigma_{h=0}^{4} c^{(h)}(x) \mathcal{L}_{h} \equiv 0$ holds in an open set, then $c^{(h)}(x)$ $\equiv 0$ for all $h$.

Proof: Suppose that the set is functionally linearly dependent. Then we can express one of the quadratic parts of the constants of the motion $\hat{\mathcal{L}}_{0}$ as a linear combination of a linearly independent subset $\left\{\hat{\mathcal{L}}_{1}, \ldots, \hat{\mathcal{L}}_{r}, 1 \leqslant r \leqslant 4\right\}$, 


$$
\hat{\mathcal{L}}_{0}=\sum_{\ell=1}^{r} c^{(\ell)}(x) \hat{\mathcal{L}}_{\ell} .
$$

Taking the Poisson bracket of both sides of this equation with $\left(p_{1}^{2}+p_{2}^{2}+p_{3}^{3}\right) / \lambda$ and using the fact that each of the $\hat{\mathcal{S}}_{h}$ is a constant of the motion, we obtain the identity

$$
\sum_{\ell=1}^{r} \sum_{i, j=1}^{3}\left(\partial_{x_{k}} c^{(\ell)}\right) a_{(\ell)}^{i j} p_{i} p_{j} p_{k}=0 .
$$

It is straightforward to check that this identity can be satisfied if and only if

$$
\sum_{\ell=1}^{r}\left(\partial_{x_{k}} c^{(\ell)}\right) a_{(\ell)}^{i j}=0, \quad 1 \leqslant i, j, k \leqslant 3
$$

Since the set $\left\{\hat{\mathcal{L}}_{1}, \ldots, \hat{\mathcal{L}}_{r}\right\}$, is linearly independent, we have $\partial_{x_{k}} c^{(\ell)} \equiv 0$ for $1 \leqslant k \leqslant 3,1 \leqslant \ell \leqslant r$. Hence the $c^{(\ell)}$ are constants, which means that

$$
\hat{\mathcal{L}}_{0}-\sum_{\ell=1}^{r} c^{(\ell)} \hat{\mathcal{L}}_{\ell}=c,
$$

where $c$ is a constant. Thus the set $\left\{\mathcal{H}_{0}, \mathcal{L}_{1}, \ldots, \mathcal{L}_{4}\right\}$ is functionally dependent. This is a contradiction.

Q.E.D.

Corollary 1: The weakly functionally independent set $\left\{\mathcal{H}=\mathcal{S}_{0}, \mathcal{S}_{1}, \ldots, \mathcal{S}_{4}\right\}$ is also functionally linearly independent in the sense that if the relation $\Sigma_{h=0}^{4} c^{(h)}(x) \mathcal{S}_{h} \equiv 0$ holds in an open set, then $c^{(h)}(x) \equiv 0$ for all $h$.

Proof: Suppose that the set is functionally linearly dependent. Then we can express one of the constants of the motion $\hat{\mathcal{S}}_{0^{\prime}}$ as a linear combination of a linearly independent subset $\left\{\hat{\mathcal{S}}_{1}, \ldots, \hat{\mathcal{L}}_{r}, 1 \leqslant r \leqslant 4\right\}$

$$
\hat{\mathcal{S}}_{0}=\sum_{\ell=1}^{r} c^{(\ell)}(x) \hat{\mathcal{S}}_{\ell}
$$

Taking the Poisson bracket of both sides of this equation with $\left(p_{1}^{2}+p_{2}^{2}+p_{3}^{3}\right) / \lambda+V$ and using the fact that each of the $\hat{\mathcal{S}}_{h}$ is a constant of the motion, we obtain the identities

$$
\sum_{\ell=1}^{r}\left(\partial_{x_{k}} c^{(\ell)}\right) a_{(\ell)}^{i j}=0, \sum_{\ell=1}^{r} \partial_{x_{k}} c^{(\ell)} W_{(\ell)}, \quad 1 \leqslant i, j, k \leqslant 3 .
$$

Since the set $\left\{\hat{\mathcal{S}}_{1}, \ldots, \hat{\mathcal{S}}_{r}\right\}$, is functionally linearly independent, we have $\partial_{x_{k}} c^{(\ell)} \equiv 0$ for $1 \leqslant k$ $\leqslant 3,1 \leqslant \ell \leqslant r$. Hence the $c^{(\ell)}$ are constants, which means that

$$
\hat{\mathcal{S}}_{0}-\sum_{\ell=1}^{r} c^{(\ell)} \hat{\mathcal{S}}_{\ell}=0 .
$$

Thus the set $\left\{\mathcal{S}_{0}, \ldots, \mathcal{S}_{4}\right\}$ is functionally dependent. This is a contradiction.

Q.E.D.

We can write the system of Bertrand-Darboux equations in the matrix form $C v=\widetilde{v}^{(1)} V_{1}$ $+\widetilde{v}^{(2)} V_{2}+\widetilde{v}^{(3)} V_{3}$, or 


$$
\begin{aligned}
& \left(\begin{array}{ccccc}
0 & a^{12} & a^{11}-a^{22} & a^{31} & -a^{32} \\
a^{13} & 0 & -a^{23} & a^{21} & a^{11}-a^{33} \\
a^{32} & -a^{32} & -a^{13} & a^{22}-a^{33} & a^{12}
\end{array}\right)\left(\begin{array}{c}
V_{33}-V_{11} \\
V_{22}-V_{11} \\
V_{12} \\
V_{32} \\
V_{31}
\end{array}\right) \\
& \quad=\frac{1}{\lambda}\left(\begin{array}{c}
\left(\lambda a^{12}\right)_{1}-\left(\lambda a^{11}\right)_{2} \\
\left(\lambda a^{31}\right)_{1}-\left(\lambda a^{11}\right)_{3} \\
\left(\lambda a^{31}\right)_{2}-\left(\lambda a^{21}\right)_{3}
\end{array}\right) V_{1}+\frac{1}{\lambda}\left(\begin{array}{c}
\left(\lambda a^{22}\right)_{1}-\left(\lambda a^{21}\right)_{2} \\
\left(\lambda a^{32}\right)_{1}-\left(\lambda a^{12}\right)_{3} \\
\left(\lambda a^{32}\right)_{2}-\left(\lambda a^{22}\right)_{3}
\end{array}\right) V_{2}+\frac{1}{\lambda}\left(\begin{array}{c}
\left(\lambda a^{32}\right)_{1}-\left(\lambda a^{31}\right)_{2} \\
\left(\lambda a^{33}\right)_{1}-\left(\lambda a^{13}\right)_{3} \\
\left(\lambda a^{33}\right)_{2}-\left(\lambda a^{23}\right)_{3}
\end{array}\right) V_{3} .
\end{aligned}
$$

Corollary 2: Suppose the set $\left\{\mathcal{H}, \mathcal{S}_{1}, \ldots, \mathcal{S}_{4}\right\}$ is functionally independent. Then for general $x$ the $4 \times 5$ matrix

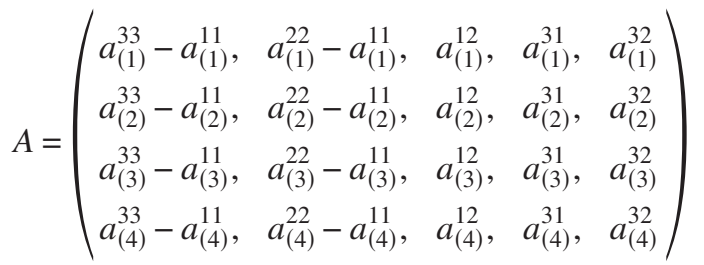

has rank 4 , where the functions $a_{(h)}^{i j}(\mathbf{x})$ are given by (11).

There are four sets of equations (13), one for each of the functionally independent symmetries (in addition to the Hamiltonian). We can write them as a single matrix equation $B v=b$ where $B$ is $12 \times 5, b$ is $12 \times 1$ and

$$
v=\left(\begin{array}{c}
V_{33}-V_{11} \\
V_{22}-V_{11} \\
V_{12} \\
V_{32} \\
V_{31}
\end{array}\right)
$$

Lemma 1: If the set $\left\{\mathcal{H}, \mathcal{S}_{1}, \ldots, \mathcal{S}_{4}\right\}$ is functionally independent, the matrix $B$ has rank 5.

Proof: In the neighborhood of a general point $\left(x_{0}, y_{0}, z_{0}\right)$ the matrix $A$ of Corollary 2 has rank 4. Thus the possible reduced row equivalence forms (RREF) for $A$ at $\left(x_{0}, y_{0}, z_{0}\right)$ are

$$
\begin{gathered}
\text { I }\left(\begin{array}{lllll}
1, & 0, & 0, & 0, & \alpha \\
0, & 1, & 0, & 0, & \beta \\
0, & 0, & 1, & 0, & \gamma \\
0, & 0, & 0, & 1, & \delta
\end{array}\right) ; \quad \text { II }\left(\begin{array}{ccccc}
1, & 0, & 0, & \alpha, & 0 \\
0, & 1, & 0, & \beta, & 0 \\
0, & 0, & 1, & \gamma, & 0 \\
0, & 0, & 0, & 0, & 1
\end{array}\right) ; \quad \text { III }\left(\begin{array}{ccccc}
1, & 0, & \alpha, & 0, & 0 \\
0, & 1, & \beta, & 0, & 0 \\
0, & 0, & 0, & 1, & 0 \\
0, & 0, & 0, & 0, & 1
\end{array}\right) ; \\
\operatorname{IV}\left(\begin{array}{ccccc}
1, & \alpha, & 0, & 0, & 0 \\
0, & 0, & 1, & 0, & 0 \\
0, & 0, & 0, & 1, & 0 \\
0, & 0, & 0, & 0, & 1
\end{array}\right) ; \quad \mathrm{V}\left(\begin{array}{lllll}
0, & 1, & 0, & 0, & 0 \\
0, & 0, & 1, & 0, & 0 \\
0, & 0, & 0, & 1, & 0 \\
0, & 0, & 0, & 0, & 1
\end{array}\right) .
\end{gathered}
$$

For each canonical form it is straightforward to check that the associated $12 \times 5$ matrix $B$ has rank 5 .

By choosing a rank 5 minor of $B$ we can solve for $v$ and obtain a solution of the form

$$
\begin{aligned}
& V_{22}=V_{11}+A^{22} V_{1}+B^{22} V_{2}+C^{22} V_{3}, \\
& V_{33}=V_{11}+A^{33} V_{1}+B^{33} V_{2}+C^{33} V_{3},
\end{aligned}
$$




$$
\begin{aligned}
& V_{12}=A^{12} V_{1}+B^{12} V_{2}+C^{12} V_{3}, \\
& V_{13}=A^{13} V_{1}+B^{13} V_{2}+C^{13} V_{3}, \\
& V_{23}=A^{23} V_{1}+B^{23} V_{2}+C^{23} V_{3} .
\end{aligned}
$$

If the augmented matrix $(B, b)$ has rank $r^{\prime}>r$ then there will be $r^{\prime}-r$ additional conditions of the form $D_{(s)}^{1} V_{1}+D_{(s)}^{2} V_{2}+D_{(s)}^{3} V_{3}=0, s=1, \ldots, r^{\prime}-r$. Here the $A^{i j}, B^{i j}, C^{i j}, D_{(s)}^{i}$ are functions of $x$ that can be calculated explicitly. For convenience we take $A^{i j} \equiv A^{j i}, B^{i j} \equiv B^{j i}, C^{i j} \equiv C^{j i}$.

Suppose now that the superintegrable system is such that $r^{\prime}=r$ so that relations (14) are equivalent to $B v=b$. Further, suppose the integrability conditions for system (14) are satisfied identically. In this case we say that the potential is nondegenerate. Otherwise the potential is degenerate. If $V$ is nondegenerate then at any point $\mathbf{x}_{0}$, where the $A^{i j}, B^{i j}, C^{i j}$ are defined and analytic, there is a unique solution $V(\mathbf{x})$ arbitrarily prescribed values of $V_{1}\left(\mathbf{x}_{0}\right), V_{2}\left(\mathbf{x}_{0}\right), V_{3}\left(\mathbf{x}_{0}\right), V_{11}\left(\mathbf{x}_{0}\right)$ [as well as the value of $V\left(\mathbf{x}_{0}\right)$ itself]. The points $\mathbf{x}_{0}$ are called regular. The points of singularity for the $A^{i j}, B^{i j}, C^{i j}$ form a manifold of dimension $<3$. Degenerate potentials depend on fewer parameters. For example, we could have $r^{\prime}=r$ but the integrability conditions are not satisfied identically. This occurs for the generalized Kepler-Coulomb potential where the integrability conditions lead to an additional equation of the form $V_{11}=A^{11} V_{1}+B^{11} V_{2}$ $+C^{11} V_{3}$ so that $V_{11}$ cannot be prescribed arbitrarily.

From this point on we assume that $V$ is nondegenerate. Substituting the requirement for a nondegenerate potential (14) into the Bertrand-Darboux equations (10) we obtain three equations for the derivatives $a_{i}^{j k}$, the first of which is

$$
\begin{aligned}
\left(a_{3}^{11}-\right. & \left.a_{1}^{31}\right) V_{1}+\left(a_{3}^{12}-a_{1}^{32}\right) V_{2}+\left(a_{3}^{13}-a_{1}^{33}\right) V_{3}+a^{12}\left(A^{23} V_{1}+B^{23} V_{2}+C^{23} V_{3}\right)-\left(a^{33}-a^{11}\right) \\
& \times\left(A^{13} V_{1}+B^{13} V_{2}+C^{13} V_{3}\right)-a^{23}\left(A^{12} V_{1}+B^{12} V_{2}+C^{12} V_{3}\right)+a^{13}\left(A^{33} V_{1}+B^{33} V_{2}+C^{33} V_{3}\right) \\
= & \left(-G_{3} a^{11}+G_{1} a^{13}\right) V_{1}+\left(-G_{3} a^{12}+G_{1} a^{23}\right) V_{2}+\left(-G_{3} a^{13}+G_{1} a^{33}\right) V_{3},
\end{aligned}
$$

and the other two are obtained in a similar fashion.

Since $V$ is nondegenerate we can compute all of the third partial derivatives of $V$. In fact, differentiating each of the equations (14) with respect to $x_{j}, j=1,2,3$ we obtain 15 equations for the 10 distinct partial derivatives $V_{i j k}$. For example,

$$
\begin{aligned}
& V_{123}= \partial_{2} V_{13}=A_{2}^{13} V_{1}+B_{2}^{13} V_{2}+C_{2}^{13} V_{3}+A^{13} V_{12}+B^{13} V_{22}+C^{13} V_{23} \\
&= \partial_{1} V_{23}=A_{1}^{23} V_{1}+B_{1}^{23} V_{2}+C_{1}^{23} V_{3}+A^{23} V_{11}+B^{23} V_{12}+C^{23} V_{13} \\
&= \partial_{3} V_{12}=A_{3}^{12} V_{1}+B_{3}^{12} V_{2}+C_{3}^{12} V_{3}+A^{12} V_{13}+B^{12} V_{23}+C^{12} V_{33}, \\
& V_{111}=\left(A_{2}^{12}-A_{1}^{22}\right) V_{1}+\left(B_{2}^{12}-B_{1}^{22}\right) V_{2}+\left(C_{2}^{12}-C_{1}^{22}\right) V_{3}-A^{22} V_{11} \\
&+B^{12} V_{22}+\left(A^{12}-B^{22}\right) V_{12}-C^{22} V_{13}+C^{12} V_{23} \\
&=\left(A_{3}^{13}-A_{1}^{33}\right) V_{1}+\left(B_{3}^{13}-B_{1}^{33}\right) V_{2}+\left(C_{3}^{13}-C_{1}^{33}\right) V_{3}-A^{33} V_{11} \\
&+C^{13} V_{33}+\left(A^{13}-C^{33}\right) V_{13}-B^{33} V_{12}+B^{13} V_{23}, \\
& V_{223}=\left(A_{1}^{31}+A_{3}^{22}\right) V_{1}+\left(B_{1}^{31}+B_{3}^{22}\right) V_{2}+\left(C_{1}^{31}+C_{3}^{22}\right) V_{3}+A^{31} V_{11}+C^{22} V_{33}+B^{13} V_{21}+\left(C^{31}+A^{22}\right) V_{13} \\
&+B^{22} V_{23}=A_{2}^{23} V_{1}+B_{2}^{23} V_{2}+C_{2}^{23} V_{3}+A^{23} V_{12}+C^{23} V_{32}+B^{23} V_{22},
\end{aligned}
$$




$$
\begin{aligned}
V_{332}= & \left(A_{1}^{12}+A_{2}^{33}\right) V_{1}+\left(B_{1}^{12}+B_{3}^{33}\right) V_{2}+\left(C_{1}^{12}+C_{2}^{33}\right) V_{3}+A^{12} V_{11}+B^{33} V_{22}+C^{12} V_{31}+\left(B^{12}+A^{33}\right) V_{12} \\
& +C^{33} V_{23}=A_{3}^{23} V_{1}+B_{3}^{23} V_{2}+C_{3}^{23} V_{3}+A^{23} V_{13}+B^{23} V_{23}+C^{23} V_{33},
\end{aligned}
$$

with analogous expressions for the other third derivatives. Similarly all higher order derivatives of $V$ can be computed from these. The right-hand side of each of these equations can be expressed as an explicit linear combination of $V_{1}, V_{2}, V_{3}, V_{11}$ with analytic functions of $x_{1}, x_{2}, x_{3}$ as coefficients. Thus if the potential $V$ belongs to the solution space then $V$ can depend on at most four parameters, in addition to a trivial additive constant. We can choose these parameters to be $V_{1}\left(x_{0}, y_{0}, z_{0}\right), V_{2}\left(x_{0}, y_{0}, z_{0}\right), V_{3}\left(x_{0}, y_{0}, z_{0}\right), V_{11}\left(x_{0}, y_{0}, z_{0}\right)$ for any fixed regular point $\left(x_{0}, y_{0}, z_{0}\right)$. Then all higher derivatives can be computed by successive differentiation of relations (14). Thus our potential is nondegenerate, i.e., it depends non trivially on these four arbitrary parameters, so that all higher-order integrability conditions are satisfied.

Then, equating coefficients of $V_{1}, V_{2}, V_{3}, V_{11}$ on each side of the conditions $\partial_{1} V_{23}=\partial_{2} V_{13}$ $=\partial_{3} V_{12}, \partial_{3} V_{23}=\partial_{2} V_{33}$, etc., we obtain integrability conditions, the simplest of which include

$$
\begin{gathered}
A^{23}=B^{13}=C^{12}, \quad B^{12}-A^{22}=C^{13}-A^{33}, \\
B^{23}=A^{31}+C^{22}, \quad C^{23}=A^{12}+B^{33}, \\
A_{1}^{12}+B^{12} A^{12}+A_{2}^{33}+A^{33} A^{12}+B^{33} A^{22}+C^{33} A^{23}=A_{3}^{23}+B^{23} A^{23}+C^{23} A^{33}, \\
A_{2}^{13}+A^{13} A^{12}+B^{13} A^{22}+C^{13} A^{23}=A_{1}^{23}+B^{23} A^{12}+C^{23} A^{13}=A_{3}^{12}+A^{13} A^{12}+B^{12} A^{23}+C^{12} A^{33} .
\end{gathered}
$$

All of these conditions, analytic expressions in $x, y, z$, must hold identically in a common domain to have a nondegenerate system. Note that if $r^{\prime}>r$ then there will be conditions relating the parameters $V_{1}\left(x_{0}, y_{0}, z_{0}\right), V_{2}\left(x_{0}, y_{0}, z_{0}\right), V_{3}\left(x_{0}, y_{0}, z_{0}\right), V_{11}\left(x_{0}, y_{0}, z_{0}\right)$, so we cannot have a nondegenerate system in that case.

We can further clarify the situation by introducing the dependent variables $W^{(1)}=V_{1}, W^{(2)}$ $=V_{2}, W^{(3)}=V_{3}, W^{(4)}=V_{11}$, the vector

$$
\mathbf{w}=\left(\begin{array}{l}
W^{(1)} \\
W^{(2)} \\
W^{(3)} \\
W^{(4)}
\end{array}\right),
$$

and the matrices

$$
\begin{gathered}
\mathbf{A}^{(1)}=\left(\begin{array}{cccc}
0 & 0 & 0 & 1 \\
A^{12} & B^{12} & C^{12} & 0 \\
A^{13} & B^{13} & C^{13} & 0 \\
A^{14} & B^{14} & C^{14} & B^{12}-A^{22}
\end{array}\right), \\
\mathbf{A}^{(2)}=\left(\begin{array}{llll}
A^{12} & B^{12} & C^{12} & 0 \\
A^{22} & B^{22} & C^{22} & 1 \\
A^{23} & B^{23} & C^{23} & 0 \\
A^{24} & B^{24} & C^{24} & A^{12}
\end{array}\right),
\end{gathered}
$$




$$
\mathbf{A}^{(3)}=\left(\begin{array}{cccc}
A^{13} & B^{13} & C^{13} & 0 \\
A^{23} & B^{23} & C^{23} & 0 \\
A^{33} & B^{33} & C^{33} & 1 \\
A^{34} & B^{34} & C^{34} & A^{13}
\end{array}\right),
$$

where

$$
\begin{aligned}
& A^{14}=A_{2}^{12}-A_{1}^{22}+B^{12} A^{22}+A^{12} A^{12}-B^{22} A^{12}-C^{22} A^{13}+C^{12} A^{23} \\
& =A_{3}^{13}-A_{1}^{33}+B^{13} A^{23}+A^{13} A^{13}-B^{33} A^{12}-C^{33} A^{13}+C^{13} A^{33}, \\
& B^{14}=B_{2}^{12}-B_{1}^{22}+B^{12} B^{22}+A^{12} B^{12}-B^{22} B^{12}-C^{22} B^{13}+C^{12} B^{23} \\
& =B_{3}^{13}-B_{1}^{33}+B^{13} B^{23}+A^{13} B^{13}-B^{33} B^{12}-C^{33} B^{13}+C^{13} B^{33}, \\
& C^{14}=C_{2}^{12}-C_{1}^{22}+B^{12} C^{22}+A^{12} C^{12}-B^{22} C^{12}-C^{22} C^{13}+C^{12} C^{23} \\
& =C_{3}^{13}-C_{1}^{33}+B^{13} C^{23}+A^{13} C^{13}-B^{23} C^{12}-C^{33} C^{13}+C^{13} C^{33}, \\
& A^{24}=A_{1}^{12}+B^{12} A^{12}+C^{12} A^{13}, \quad B^{24}=B_{1}^{12}+B^{12} B^{12}+C^{12} B^{13}, \\
& C^{24}=C_{1}^{12}+B^{12} C^{12}+C^{12} C^{13}, \\
& A^{34}=A_{1}^{13}+B^{13} A^{12}+C^{13} A^{13}, \quad B^{34}=B_{1}^{13}+B^{13} B^{12}+C^{13} B^{13}, \\
& C^{34}=C_{1}^{13}+B^{13} C^{12}+C^{13} C^{13} .
\end{aligned}
$$

Then the conditions (17) must hold as well as the integrability conditions for the system

$$
\partial_{x_{j}} \mathbf{w}=\mathbf{A}^{(j)} \mathbf{w}, \quad j=1,2,3 .
$$

The integrability conditions are

$$
A_{i}^{(j)}-A_{j}^{(i)}=A^{(i)} A^{(j)}-A^{(j)} A^{(i)} \equiv\left[A^{(i)}, A^{(j)}\right] .
$$

The integrability conditions (17) and (24) are analytic expressions in $x_{1}, x_{2}, x_{3}$ and must hold identically. Then the system has a solution $V$ depending on four parameters (plus an arbitrary additive parameter). For convenience in the arguments to follow we set

$$
\begin{gathered}
\mathcal{U}^{1}=A_{2}^{(3)}-A_{3}^{(2)}-\left[A^{(2)}, A^{(3)}\right], \quad \mathcal{U}^{2}=A_{3}^{(1)}-A_{1}^{(3)}-\left[A^{(3)}, A^{(1)}\right], \\
\mathcal{U}^{3}=A_{1}^{(2)}-A_{2}^{(1)}-\left[A^{(1)}, A^{(2)}\right],
\end{gathered}
$$

so that the identities are

$$
\mathcal{U}^{1}=\mathcal{U}^{2}=\mathcal{U}^{\beta}=0 .
$$

We have shown that a weakly functionally independent set of five symmetries (or constants of the motion) is functionally linearly independent. For systems with nondegenerate potentials, the converse holds.

Theorem 2: Let

$$
\mathcal{S}_{h}=\sum_{j, k=1}^{3} a_{(h)}^{j k} p_{k} p_{j}+W_{(h)}, \quad h=1, \ldots, 5, \quad \mathcal{H}=\mathcal{S}_{1}
$$


be functionally linearly independent symmetries for a system with nondegenerate potential $V$ $=W_{(1)}$. Then these symmetries are weakly functionally independent.

Proof: By assumption, the set $\left\{\mathcal{S}_{h}\right\}$ is functionally linearly independent. Suppose it is also functionally dependent. This means that the set of differentials $\left\{\mathrm{d} \mathcal{S}_{h}\right\}$ is dependent, i.e., that the $5 \times 6$ matrix

$$
\left(\begin{array}{cccccc}
\sum_{i} a_{(1)}^{i 1} p_{i} & \sum_{i} a_{(1)}^{i 2} p_{i} & \sum_{i} a_{(1)}^{i 3} p_{i} & K_{14}+\sum_{i} a_{(1)}^{j 1} V_{j} & K_{15}+\sum_{i} a_{(1)}^{j 2} V_{j} & K_{16}+\sum_{i} a_{(1)}^{j 3} V_{j} \\
\vdots & \vdots & \vdots & \vdots & \vdots & \vdots \\
\sum_{i} a_{(5)}^{i 1} p_{i} & \sum_{i} a_{(5)}^{i 2} p_{i} & \sum_{i} a_{(5)}^{i 3} p_{i} & K_{54}+\sum_{i} a_{(5)}^{j 1} V_{j} & K_{55}+\sum_{i} a_{(5)}^{j 2} V_{j} & K_{56}+\sum_{i} a_{(5)}^{j 3} V_{j}
\end{array}\right),
$$

where

$$
K_{\ell, 3+s}=\sum_{i j} a_{(\ell), s}^{i j} p_{i} p_{j}
$$

is of rank $<5$ for all values of $p_{j}, V_{j}$. Thus all $5 \times 5$ minors must vanish identically in $p_{j}, V_{j}$. It is an easy consequence of this that all $5 \times 5$ minors of the $5 \times 6$ matrix

$$
\left(\begin{array}{rrrrrr}
a_{(1)}^{11} & a_{(1)}^{12} & a_{(1)}^{13} & a_{(1)}^{22} & a_{(1)}^{23} & a_{(1)}^{33} \\
\vdots & \vdots & \vdots & \vdots & \vdots & \vdots \\
a_{(5)}^{11} & a_{(5)}^{12} & a_{(5)}^{13} & a_{(5)}^{22} & a_{(5)}^{23} & a_{(5)}^{33}
\end{array}\right)
$$

vanish, hence that this matrix have rank $<5$. Thus $\left\{\mathcal{L}_{h}: h=1, \ldots, 5\right\}$ is functionally linearly dependent. Contradiction.

Q.E.D.

Since (as we assume) the potential is nondegenerate, at any regular point $\mathbf{x}_{0}$ the first derivatives $V_{1}, V_{2}, V_{3}$ can be chosen arbitrarily. Thus the coefficients of $V_{j}$ on both sides of equation (13) must be equal. From this, we obtain the relations

$$
\begin{aligned}
& a_{3}^{11}-a_{1}^{31}=-a^{12} A^{23}+\left(a^{33}-a^{11}\right) A^{13}+a^{23} A^{12}-a^{13} A^{33}-G_{3} a^{11}+G_{1} a^{13}, \\
& a_{3}^{12}-a_{1}^{32}=-a^{12} B^{23}+\left(a^{33}-a^{11}\right) B^{13}+a^{23} B^{12}-a^{13} B^{33}-G_{3} a^{12}+G_{1} a^{23}, \\
& a_{3}^{13}-a_{1}^{33}=-a^{12} C^{23}+\left(a^{33}-a^{11}\right) C^{13}+a^{23} C^{12}-a^{13} C^{33}-G_{3} a^{13}+G_{1} a^{33},
\end{aligned}
$$

with six analogous relations from the other two Bertrand-Darboux equations. Using these nine relations and Eqs. (8) we can solve for all of the first partial derivatives $a_{i}^{j k}$ to obtain

$$
\begin{gathered}
a_{1}^{11}=-G_{1} a^{11}-G_{2} a^{12}-G_{3} a^{13}, \\
a_{2}^{22}=-G_{1} a^{12}-G_{2} a^{22}-G_{3} a^{23}, \\
a_{3}^{33}=-G_{1} a^{13}-G_{2} a^{23}-G_{3} a^{33}, \\
3 a_{2}^{12}=a^{12} A^{22}-\left(a^{22}-a^{11}\right) A^{12}-a^{23} A^{13}+a^{13} A^{23}+G_{2} a^{11}-2 G_{1} a^{12}-G_{2} a^{22}-G_{3} a^{23}, \\
3 a_{2}^{11}=-2 a^{12} A^{22}+2\left(a^{22}-a^{11}\right) A^{12}+2 a^{23} A^{13}-2 a^{13} A^{23}-2 G_{2} a^{11}+G_{1} a^{12}-G_{2} a^{22}-G_{3} a^{23}, \\
3 a_{3}^{13}=-a^{12} C^{23}+\left(a^{33}-a^{11}\right) C^{13}+a^{23} C^{12}-a^{13} C^{33}-G_{1} a^{11}-G_{2} a^{12}-2 G_{3} a^{13}+G_{1} a^{33}, \\
3 a_{1}^{33}=2 a^{12} C^{23}-2\left(a^{33}-a^{11}\right) C^{13}-2 a^{23} C^{12}+2 a^{13} C^{33}-G_{1} a^{11}-G_{2} a^{12}+G_{3} a^{13}-2 G_{1} a^{33},
\end{gathered}
$$




$$
\begin{array}{rl}
3 a_{2}^{23}= & a^{23}\left(B^{33}-B^{22}\right)-\left(a^{33}-a^{22}\right) B^{23}-a^{13} B^{12}+a^{12} B^{13}-G_{1} a^{13}-2 G_{2} a^{23}-G_{3} a^{33}+G_{3} a^{22}, \\
3 a_{3}^{22}= & -2 a^{23}\left(B^{33}-B^{22}\right)+2\left(a^{33}-a^{22}\right) B^{23}+2 a^{13} B^{12}-2 a^{12} B^{13}-G_{1} a^{13}+G_{2} a^{23}-G_{3} a^{33}-2 G_{3} a^{22}, \\
3 & a_{1}^{13}=-a^{23} A^{12}+\left(a^{11}-a^{33}\right) A^{13}+a^{13} A^{33}+a^{12} A^{23}-2 G_{1} a^{13}-G_{2} a^{23}-G_{3} a^{33}+G_{3} a^{11}, \\
3 & a_{3}^{11}=2 a^{23} A^{12}+2\left(a^{33}-a^{11}\right) A^{13}-2 a^{13} A^{33}-2 a^{12} A^{23}+G_{1} a^{13}-G_{2} a^{23}-G_{3} a^{33}-2 G_{3} a^{11}, \\
3 a_{2}^{33}= & -2 a^{13} C^{12}+2\left(a^{22}-a^{33}\right) C^{23}+2 a^{12} C^{13}-2 a^{23}\left(C^{22}-C^{33}\right)-G_{1} a^{12}-G_{2} a^{22}+G_{3} a^{23}-2 G_{2} a^{33}, \\
3 a_{3}^{23}= & a^{13} C^{12}-\left(a^{22}-a^{33}\right) C^{23}-a^{12} C^{13}-a^{23}\left(C^{33}-C^{22}\right)-G_{1} a^{12}-G_{2} a^{22}-2 G_{3} a^{23}+G_{2} a^{33}, \\
3 & 3 a_{2}^{12}=-a^{13} B^{23}+\left(a^{22}-a^{11}\right) B^{12}-a^{12} B^{22}+a^{23} B^{13}-G_{1} a^{11}-2 G_{2} a^{12}-G_{3} a^{13}+G_{1} a^{22}, \\
3 a_{1}^{22}= & 2 a^{13} B^{23}-2\left(a^{22}-a^{11}\right) B^{12}+2 a^{12} B^{22}-2 a^{23} B^{13}-G_{1} a^{11}+G_{2} a^{12}-G_{3} a^{13}-2 G_{1} a^{22}, \\
3 a_{1}^{23}= & a^{12}\left(B^{23}+C^{22}\right)+a^{11}\left(B^{13}+C^{12}\right)-a^{22} C^{12}-a^{33} B^{13}+a^{13}\left(B^{33}+C^{23}\right)-a^{23}\left(C^{13}+B^{12}\right) \\
& -2 G_{1} a^{23}+G_{2} a^{13}+G_{3} a^{12}, \\
3 & a_{3}^{12}=a^{12}\left(-2 B^{23}+C^{22}\right)+a^{11}\left(C^{12}-2 B^{13}\right)-a^{22} C^{12}+2 a^{33} B^{13}+a^{13}\left(-2 B^{33}+C^{23}\right) \\
& +a^{23}\left(-C^{13}+2 B^{12}\right)-2 G_{3} a^{12}+G_{2} a^{13}+G_{1} a^{23}, \\
3 a_{2}^{13}= & a^{12}\left(B^{23}-2 C^{22}\right)+a^{11}\left(B^{13}-2 C^{12}\right)+2 a^{22} C^{12}-a^{33} B^{13}+a^{13}\left(B^{33}-2 C^{23}\right)+a^{23}\left(2 C^{13}-B^{12}\right) \\
& -2 G_{2} a^{13}+G_{1} a^{23}+G_{3} a^{12},
\end{array}
$$

plus the linear relations

$$
\begin{gathered}
A^{23}=B^{13}=C^{12}, \quad B^{23}-A^{13}-C^{22}=0, \\
B^{12}-A^{22}+A^{33}-C^{13}=0, \quad B^{33}+A^{12}-C^{23}=0 .
\end{gathered}
$$

Using the linear relations we can express $C^{12}, C^{13}, C^{22}, C^{23}$, and $B^{13}$ in terms of the remaining 10 functions.

Since the above system of first-order partial differential equations is involutive the general solution for the six functions $a^{j k}$ can depend on at most six parameters, the values $a^{j k}\left(\mathbf{x}_{0}\right)$ at a fixed regular point $\mathbf{x}_{0}$. For the integrability conditions we define the vector-valued function

$$
\mathbf{h}(x, y, z)=\left(\begin{array}{l}
a^{11} \\
a^{12} \\
a^{13} \\
a^{22} \\
a^{23} \\
a^{33}
\end{array}\right)
$$

and directly compute the $6 \times 6$ matrix functions $\mathcal{A}^{(j)}$ to get the first-order system

$$
\partial_{x_{j}} \mathbf{h}=\mathcal{A}^{(j)} \mathbf{h}, \quad j=1,2,3 .
$$

The integrability conditions for this system are are 


$$
\mathcal{A}_{i}^{(j)} \mathbf{h}-\mathcal{A}_{j}^{(i)} \mathbf{h}=\mathcal{A}^{(i)} \mathcal{A}^{(j)} \mathbf{h}-\mathcal{A}^{(j)} \mathcal{A}^{(i)} \mathbf{h} \equiv\left[\mathcal{A}^{(i)}, \mathcal{A}^{(j)}\right] \mathbf{h} .
$$

In terms of the $6 \times 6$ matrices

$$
\begin{gathered}
\mathcal{S}^{(1)}=\mathcal{A}_{2}^{(3)}-\mathcal{A}_{3}^{(2)}-\left[\mathcal{A}^{(2)}, \mathcal{A}^{(3)}\right], \quad \mathcal{S}^{(2)}=\mathcal{A}_{3}^{(1)}-\mathcal{A}_{1}^{(3)}-\left[\mathcal{A}^{(3)}, \mathcal{A}^{(1)}\right], \\
\mathcal{S}^{(3)}=\mathcal{A}_{1}^{(2)}-\mathcal{A}_{2}^{(1)}-\left[\mathcal{A}^{(1)}, \mathcal{A}^{(2)}\right],
\end{gathered}
$$

the integrabilty conditions are

$$
\mathcal{S}^{(1)} \mathbf{h}=\mathcal{S}^{(2)} \mathbf{h}=\mathcal{S}^{(3)} \mathbf{h}=0 .
$$

\section{THE $5 \Rightarrow 6$ THEOREM}

Now assume that the system of equations (27) admits a six-parameter family of solutions $a^{j k}$. (The requirement of superintegrability appears to guarantee only a five-parameter family of solutions.) Thus at any regular point we can prescribe the values of the $a^{j k}$ arbitrarily. This means that (29) and (30) holds identically in h. Thus $\mathcal{S}^{(1)}=\mathcal{S}^{(2)}=\mathcal{S}^{(3)}=0$. Using these expressions, we can perform a tedious but straightforward Maple-assisted computation that yields

(1) An expression for each of the first partial derivatives $\partial_{\ell} A^{i j}, \partial_{\ell} B^{i j}, \partial_{\ell} C^{i j}$, for the 10 independent functions as homogeneous polynomials of order at most two in the $A^{i^{\prime} j^{\prime}}, B^{i^{\prime} j^{\prime}}, C^{i^{\prime} j^{\prime}}$. There are $30=3 \times 10$ such expressions in all. An example is

$$
B_{2}^{12}=\frac{2}{3} A^{12} B^{12}-\frac{1}{6} B^{12} G_{2}-\frac{5}{6} G_{1} A^{12}-\frac{1}{6} G_{1} G_{2}+\frac{1}{3} B^{22} B^{12}+\frac{1}{3} B^{22} G_{1}+\frac{1}{3} A^{23} B^{23}-\frac{7}{6} G_{3} A^{23}+\frac{1}{2} G_{12} .
$$

(2) Exactly five quadratic identities for the 10 independent functions,

(a)

$$
\begin{aligned}
& -A^{23} B^{33}-A^{12} A^{23}+A^{13} B^{12}+B^{22} A^{23}+B^{23} A^{33}+\frac{1}{2} A^{22} G_{3}-\frac{1}{2} A^{33} G_{3}-\frac{1}{2} B^{12} G_{3}-\frac{1}{2} G_{1} G_{3} \\
& -\frac{1}{2} A^{13} G_{1}+\frac{3}{2} G_{13}-\frac{1}{2} A^{23} G_{2}-A^{22} B^{23}=0
\end{aligned}
$$

(b)

$$
\begin{aligned}
& \left(A^{33}\right)^{2}+B^{12} A^{33}-A^{33} A^{22}-B^{33} A^{12}-C^{33} A^{13}+B^{22} A^{12}-B^{12} A^{22}+A^{13} B^{23}-\left(A^{12}\right)^{2}+\frac{3}{2} G_{22} \\
& \quad-\frac{1}{2} G_{y}^{2}-\frac{3}{2} G_{33}+\frac{1}{2} A^{13} G_{3}+\frac{1}{2} B^{33} G_{2}-\frac{1}{2} A^{22} G_{1}+\frac{1}{2} A^{33} G_{1}-\frac{1}{2} B^{23} G_{3}-\frac{1}{2} B^{22} G_{2}+\frac{1}{2} C^{33} G_{3} \\
& \quad+\frac{1}{2}\left(G_{3}\right)^{2}=0
\end{aligned}
$$

(c)

$$
\begin{gathered}
-\left(B^{33}\right)^{2}-B^{33} A^{12}+B^{33} B^{22}+B^{12} A^{33}+B^{23} C^{33}-\left(B^{23}\right)^{2}+\left(B^{12}\right)^{2}+\frac{1}{2}\left(G_{1}\right)^{2}-\frac{3}{2} G_{11}+\frac{3}{2} G_{33} \\
-\frac{1}{2} B^{33} G_{2}-\frac{1}{2} A^{33} G_{1}-\frac{1}{2}\left(G_{3}\right)^{2}-\frac{1}{2} C^{33} G_{3}=0,
\end{gathered}
$$

(d)

$$
\begin{aligned}
- & B^{12} A^{23}-A^{33} A^{23}+A^{13} B^{33}+A^{12} B^{23}+\frac{3}{2} G_{23}-\frac{1}{2} A^{23} G_{1}-\frac{1}{2} A^{12} G_{3}-\frac{1}{2} B^{23} G_{2}-\frac{1}{2} G_{2} G_{3} \\
- & \frac{1}{2} B^{33} G a_{3}=0,
\end{aligned}
$$

(e)

$$
\begin{aligned}
A^{12} B^{12} & +C^{33} A^{23}-A^{23} B^{23}+B^{33} A^{22}-B^{33} A^{33}+\frac{3}{2} G_{12}-\frac{1}{2} G_{1} G_{2}-\frac{1}{2} A^{12} G_{1} \\
- & \frac{1}{2} B^{12} G_{2}-\frac{1}{2} A^{23} G_{3}=0
\end{aligned}
$$


There are no nontrivial conditions in which some derivative of $G$ is involved as a factor in each term.

Theorem $3(5 \rightarrow 6)$ : Let $V$ be a nondegenerate potential corresponding to a conformally flat space in three dimensions that is superintegrable, i.e., suppose $V$ satisfies the equations (14), where conditions (17) and (24) hold, and there are five functionally independent constants of the motion. Then the space of second-order symmetries for the Hamiltonian $\mathcal{H}=\left(p_{x}^{2}+p_{y}^{2}\right.$ $\left.+p_{z}^{2}\right) / \lambda(x, y, z)+V(x, y, z)$ (excluding multiplication by a constant) is of dimension $D=6$.

Corollary 3: If $\mathcal{H}+V$ is a superintegrable conformally flat system with nondegenerate potential, then the dimension of the space of second-order symmetries

$$
\mathcal{S}=\sum_{k, j=1}^{3} a^{k j}(x, y, z) p_{k} p_{j}+W(x, y, z)
$$

is 6. At any regular point $\left(x_{0}, y_{0}, z_{0}\right)$ and given constants $\alpha^{k j}=\alpha^{j k}$ there is exactly one symmetry $\mathcal{S}$ (up to an additive constant) such that $a^{k j}\left(x_{0}, y_{0}, z_{0}\right)=\alpha^{k j}$. Given a set of five functionally independent second-order symmetries $\mathcal{L}=\left\{\mathcal{S}_{\ell}: \ell=1, \ldots, 5\right\}$ associated with the potential, there is always a sixth second-order symmetry $\mathcal{S}_{6}$ that is functionally dependent on $\mathcal{L}$, but functionally linearly independent.

Corollary 4: The previous theorem and corollary remain true for five weakly functionally independent second-order symmetries, if the corresponding quadratic forms $\Sigma_{k, j} a_{(\ell)}^{k j} p_{k} p_{j}, 1 \leqslant \ell$ $\leqslant 5$ are functionally linearly independent.

Proof of theorem: The proof takes many steps, most of which must be carried out with computer algebra software. We give the logic behind the proof and describe the steps in order.

If there is only a five-parameter family of solutions then (30) holds only for the $\boldsymbol{h}$ that lie in a five-dimensional space. By appropriate Euclidean transformation of coordinates, if necessary, we use Gauss-Jordan elimination and show that there is a basis for the space of the form $\widetilde{\mathbf{h}}^{j}, j=1, \ldots, 5$ where

$$
\left(\widetilde{\mathbf{h}}^{1}, \widetilde{\mathbf{h}}^{2}, \widetilde{\mathbf{h}}^{3}, \widetilde{\mathbf{h}}^{4}, \widetilde{\mathbf{h}}^{5}\right)=\left(\begin{array}{lllll}
1 & 0 & 0 & 0 & 0 \\
0 & 1 & 0 & 0 & 0 \\
0 & 0 & 1 & 0 & 0 \\
0 & 0 & 0 & 1 & 0 \\
0 & 0 & 0 & 0 & 1 \\
\alpha_{1}(x, y, z) & \alpha_{2}(x, y, z) & \alpha_{3}(x, y, z) & \alpha_{4}(x, y, z) & \alpha_{5}(x, y, z)
\end{array}\right) .
$$

Here we mean that if $\mathbf{h}$ belongs to the solution space then there are unique differentiable functions $g_{j}(x, y, z)$ such that $\mathbf{h}=\sum_{j=1}^{5} g_{j} \widetilde{\mathbf{h}}^{j}$. It follows that the integrabilty conditions become

$$
\mathcal{S}_{k j}^{(\ell)}+\alpha_{j} \mathcal{S}_{k 6}^{(\ell)}=0, \quad \ell=1, \ldots, 3, \quad k=1, \ldots, 6, \quad j=1, \ldots, 5 .
$$

Further, the conditions (30) must hold. The question that we need to decide is whether the conditions (30) and (32) imply

$$
\mathcal{S}^{(1)}=\mathcal{S}^{(2)}=\mathcal{S}^{(3)}=0 .
$$

Some of the elements of the matrices $\mathcal{S}^{(i)}$ vanish identically. Indeed

$$
\mathcal{S}_{16}^{(1)}=\mathcal{S}_{26}^{(1)}=\mathcal{S}_{46}^{(1)}=\mathcal{S}_{14}^{(2)}=\mathcal{S}_{34}^{(2)}=\mathcal{S}_{64}^{(2)}=\mathcal{S}_{41}^{(3)}=\mathcal{S}_{51}^{(3)}=\mathcal{S}_{61}^{(3)} \equiv 0 .
$$

Also

$$
\begin{gathered}
\mathcal{S}_{16}^{(2)} \equiv \mathcal{S}_{15}^{(1)} \equiv-\mathcal{S}_{11}^{(2)}, \quad \mathcal{S}_{54}^{(1)} \equiv-\mathcal{S}_{23}^{(1)}, \\
\mathcal{S}_{25}^{(1)} \equiv-\mathcal{S}_{31}^{(1)}, \quad \mathcal{S}_{34}^{(1)} \equiv \mathcal{S}_{26}^{(2)}, \quad 3 \mathcal{S}_{11}^{(3)} \equiv 2 \mathcal{S}_{11}^{(1)},
\end{gathered}
$$




$$
\mathcal{S}_{44}^{(2)} \equiv-\frac{2}{3} \mathcal{S}_{22}^{(2)}, \quad \mathcal{S}_{46}^{(3)} \equiv-\mathcal{S}_{43}^{(1)} .
$$

This implies that the following conditions must hold no matter what are the values of the $\alpha_{j}$ :

$$
\begin{gathered}
\mathcal{S}_{i j}^{(1)}=0, \quad i=1,2,4,6, \quad 1 \leqslant j \leqslant 6, \quad \mathcal{S}_{31}^{(1)}=0, \\
\mathcal{S}_{1 j}^{(2)}=\mathcal{S}_{4 j}^{(3)}=0, \quad 1 \leqslant j \leqslant 6, \quad \mathcal{S}_{54}^{(1)}=0, \quad \mathcal{S}_{44}^{(2)}=0 .
\end{gathered}
$$

We will show that the identities (33), plus the identities (26) (that must always hold) suffice to prove that

$$
\mathcal{S}^{(1)}=\mathcal{S}^{(2)}=\mathcal{S}^{(3)}=0,
$$

hence that the integrabilty conditions are satisfied identically and there is a six-parameter family of symmetries. In the first step we compute all of the identities (26) and (33) and use a subset of 17 of the identities (33) and 12 of the identities (26) to solve for each of the 30 independent partial derivatives

$$
\partial_{k} A^{12}, \quad \partial_{k} A^{13}, \quad \partial_{k} A^{22}, \quad \partial_{k} A^{23}, \quad \partial_{k} A^{33}, \quad \partial_{k} B^{12}, \quad \partial_{k} B^{22}, \quad \partial_{k} B^{23}, \quad \partial_{k} B^{33}, \quad \partial_{k} C^{33}, \quad k=1,2,3,
$$

save $\partial_{z} C^{33}$ which does not occur in these expressions. In each case we obtain an expression for the derivative as a polynomial in the 10 variables $A^{12}, \ldots, C^{33}$ with coefficients in the linear and zero-order terms that involve derivatives of $G$. Then we substitute these expressions back into the remaining conditions (26) and (33). This yields a set of four independent second-order polynomial identities, a subset of the identities (31). These identities are sufficient to verify that

$$
\mathcal{S}_{36}^{(1)}=\mathcal{S}_{56}^{(1)}=\mathcal{S}_{26}^{(2)}=\mathcal{S}_{36}^{(2)}=\mathcal{S}_{46}^{(2)}=0 \text {. }
$$

By conditions (32) this immediately implies $\mathcal{S}^{(1)}=0$, and $\mathcal{S}_{j k}^{(2)}=0$ for $j=2,3,4$ and $1 \leqslant k \leqslant 6$. Substituting our expressions for the derivatives into these identities we obtain the full set of five identities (31), and can solve for $\partial_{x} C^{33}$. This set is now sufficient to verify that

$$
\mathcal{S}_{56}^{(2)}=\mathcal{S}_{66}^{(2)}=\mathcal{S}_{16}^{(3)}=\mathcal{S}_{26}^{(3)}=\mathcal{S}_{36}^{(3)}=\mathcal{S}_{56}^{(3)}=\mathcal{S}_{66}^{(3)}=0,
$$

which implies $\mathcal{S}^{(2)}=\mathcal{S}^{(3)}=0$.

Q.E.D.

\section{THIRD-ORDER CONSTANTS OF THE MOTION}

Now we investigate the space of third-order constants of the motion, assuming a nondegenerate potential. We have

$$
\mathcal{K}=\sum_{k, j, i=1}^{3} a^{k j i}(x, y, z) p_{k} p_{j} p_{i}+b^{\ell}(x, y, z) p_{\ell},
$$

which must satisfy $\{\mathcal{H}, \mathcal{K}\}=0$. Here $a^{k j i}$ is symmetric in the indices $k, j, i$.

The conditions are

$$
\begin{gathered}
a_{i}^{i i i}=-\frac{3}{2} \sum_{s} a^{s i i}(\ln \lambda)_{s}, \\
3 a_{i}^{j i i}+a_{j}^{i i i}=-3 \sum_{s} a^{s i j}(\ln \lambda)_{s}, \quad i \neq j, \\
a_{i}^{i j j}+a_{j}^{i i j}=-\frac{1}{2} \sum_{s} a^{s j j}(\ln \lambda)_{s}-\frac{1}{2} \sum_{s} a^{s i i}(\ln \lambda)_{s}, \quad i \neq j,
\end{gathered}
$$




$$
\begin{gathered}
2 a_{i}^{i j k}+a_{j}^{k i i}+a_{k}^{j i i}=-\sum_{s} a^{s j k}(\ln \lambda)_{s}, \quad i, j, k \text { distinct, } \\
b_{k}^{j}+b_{j}^{k}=3 \lambda \sum_{s} a^{s k j} V_{s}, \quad j \neq k, \quad j, k=1,2,3, \\
b_{j}^{j}=\frac{3}{2} \lambda \sum_{s} a^{s j j} V_{s}-\frac{1}{2} \sum_{s} b^{s}(\ln \lambda)_{s}, \quad j=1,2,3,
\end{gathered}
$$

and

$$
\sum_{s} b^{s} V_{s}=0
$$

The $a^{k j i}$ is just a third-order Killing tensor. We will, as usual, require the potential $V$ to be superintegrable and non degenerate. Again, as usual, we require that the highest order terms, the $a^{k j i}$ in the constant of the motion, be independent of the four independent parameters in $V$. However, the $b^{\ell}$ must depend on these parameters. We set

$$
b^{\ell}(x, y, z)=\sum_{j=1}^{3} f^{\ell, j}(x, y, z) V_{j}(x, y, z) .
$$

[Here we are excluding the purely first-order symmetries. Also, we could add a term $f^{\ell, 11}(x, y, z) V_{11}(x, y, z)$ to the preceding expression. However condition (37) implies $f^{\ell, 11} \equiv 0$.] Substituting this expression into (37) we see that

$$
f^{\ell, j}+f^{j, \ell}=0, \quad 1 \leqslant \ell, j \leqslant 3
$$

Further

$$
b_{j}^{i}=\sum_{\ell \neq 1}\left(f_{j}^{i, \ell} V_{\ell}+f^{i, \ell} V_{j \ell}\right)
$$

where the subscript $j$ denotes the partial derivative with respect to $x_{j}$. Substituting these results and expressions (14) into the defining equations (36) and equating coefficients of $V_{1}, V_{2}, V_{3}, V_{11}$, respectively, we obtain the following independent conditions $\left[G_{s} \equiv(\ln \lambda)_{s}\right]$ :

$$
\begin{gathered}
\lambda a^{111}=\frac{2}{3}\left(f^{1,2} A^{12}+f^{1,3} A^{13}\right)+\frac{1}{3} \sum_{s=1}^{3} f^{s, 1} G_{s}, \\
\lambda a^{222}=\frac{2}{3}\left(-f^{1,2} B^{12}+f^{2,3} B^{23}\right)+\frac{1}{3} \sum_{s=1}^{3} f^{s, 2} G_{s}, \\
\lambda a^{333}=\frac{2}{3}\left(-f^{1,3} C^{13}-f^{2,3} C^{23}\right)+\frac{1}{3} \sum_{s=1}^{3} f^{s, 3} G_{s}, \\
\lambda a^{112}=\frac{2}{9}\left(f^{1,2}\left(A^{22}+B^{12}\right)+2 f^{1,3} A^{23}+f^{2,3} A^{13}\right)+\frac{1}{9} \sum_{s=1}^{3} f^{s, 2} G_{s},
\end{gathered}
$$




$$
\begin{aligned}
& \lambda a^{113}=\frac{2}{9}\left(2 f^{1,2} A^{23}+f^{1,3}\left(A^{33}+C^{13}\right)-f^{2,3} A^{12}\right)+\frac{1}{9} \sum_{s=1}^{3} f^{s, 3} G_{s}, \\
& \lambda a^{122}=\frac{2}{9}\left(f^{1,2}\left(-A^{12}+B^{22}\right)+f^{1,3} B^{23}+2 f^{2,3} A^{23}\right)+\frac{1}{9} \sum_{s=1}^{3} f^{s, 1} G_{s}, \\
& \lambda a^{223}=\frac{2}{9}\left(-2 f^{1,2} A^{23}-f^{1,3} B^{12}+f^{2,3}\left(-B^{22}+B^{33}+C^{23}\right)\right)+\frac{1}{9} \sum_{s=1}^{3} f^{s, 3} G_{s}, \\
& \lambda a^{133}=\frac{2}{9}\left(f^{1,2} C^{23}+f^{1,3}\left(-A^{13}+C^{33}\right)-2 f^{2,3} A^{23}\right)+\frac{1}{9} \sum_{s=1}^{3} f^{s, 1} G_{s}, \\
& \lambda a^{233}=\frac{2}{9}\left(-f^{1,2} C^{13}-2 f^{1,3} A^{23}+f^{2,3}\left(-B^{23}-C^{22}+C^{33}\right)\right)+\frac{1}{9} \sum_{s=1}^{3} f^{s, 2} G_{s}, \\
& \lambda a^{123}=\frac{2}{9}\left(f^{1,2} C^{22}+f^{1,3} B^{33}+f^{2,3}\left(-B^{12}+C^{13}\right)\right), \\
& f_{1}^{1,2}=\frac{1}{3}\left(f^{1,2}\left(A^{22}-2 B^{12}\right)-f^{1,3} A^{23}+f^{2,3} A^{13}\right)-\frac{1}{3} \sum_{s=1}^{3} f^{s, 2} G_{s}, \\
& f_{2}^{1,2}=\frac{1}{3}\left(f^{1,2}\left(-2 A^{12}-B^{22}\right)-f^{1,3} B^{23}+f^{2,3} A^{23}\right)+\frac{1}{3} \sum_{s=1}^{3} f^{s, 1} G_{s}, \\
& f_{1}^{1,3}=\frac{1}{3}\left(-f^{1,2} A^{23}+f^{1,3}\left(A^{33}-2 C^{13}\right)-f^{2,3} A^{12}\right)-\frac{1}{3} \sum_{s=1}^{3} f^{s, 3} G_{s}, \\
& f_{3}^{1,3}=\frac{1}{3}\left(-f^{1,2} C^{23}+f^{1,3}\left(-2 A^{13}-C^{33}\right)-f^{2,3} A^{23}\right)+\frac{1}{3} \sum_{s=1}^{3} f^{s, 1} G_{s}, \\
& f_{2}^{2,3}=\frac{1}{3}\left(f^{1,2} A^{23}-f^{1,3} B^{12}+f^{2,3}\left(-B^{22}+B^{33}-2 C^{23}\right)\right)-\frac{1}{3} \sum_{s=1}^{3} f^{s, 3} G_{s}, \\
& f_{3}^{2,3}=\frac{1}{3}\left(f^{1,2} C^{13}-f^{1,3} A^{23}+f^{2,3}\left(-2 B^{23}+C^{22}-C^{33}\right)\right)+\frac{1}{3} \sum_{s=1}^{3} f^{s, 2} G_{s},
\end{aligned}
$$

and

$$
\begin{aligned}
& f_{1}^{2,3}+f_{2}^{1,3}=\frac{1}{3}\left(-f^{1,2} C^{22}+f^{1,3}\left(2 B^{33}-3 C^{23}\right)-f^{2,3}\left(2 B^{12}+C^{13}\right)\right), \\
& -f_{1}^{2,3}+f_{3}^{1,2}=\frac{1}{3}\left(-f^{1,2}\left(2 A^{13}+B^{23}\right)-f^{1,3} B^{33}+f^{2,3}\left(B^{12}+2 C^{13}\right)\right) .
\end{aligned}
$$


We have eight equations for the nine derivatives $f_{k}^{i, j}$, and by differentiating these we have 18 independent conditions for the 18 second derivatives $f_{k \ell}^{i, j}$. Thus the system closes. A solution is determined by 12 parameters $f_{k}^{i, j}\left(\mathbf{x}_{0}\right), f_{k \ell}^{i, j}\left(\mathbf{x}_{0}\right)$ at a regular point, and these parameters are constrained by at least eight linearly independent conditions. Thus the solution space, which is obviously of dimension $\geqslant 3$ must be of dimension $\leqslant 4$. We must still apply the conditions that the $a^{i j k}$ are third-order Killing tensors.

Theorem 4: Let $\mathcal{K}$ be a third-order constant of the motion for a conformally flat superintegrable system with nondegenerate potential $V$,

$$
\mathcal{K}=\sum_{k, j, i=1}^{3} a^{k j i}(x, y, z) p_{k} p_{j} p_{i}+\sum_{\ell=1}^{3} b^{\ell}(x, y, z) p_{\ell} .
$$

Then

$$
b^{\ell}(x, y, z)=\sum_{j=1}^{3} f^{\ell, j}(x, y, z) V_{j}(x, y, z)
$$

with $f^{\ell, j}+f^{j, \ell}=0,1 \leqslant \ell, j \leqslant 3$. The $a^{i j k}, b^{\ell}$ are uniquely determined by the four numbers

$$
f^{1,2}\left(x_{0}, y_{0}, z_{0}\right), \quad f^{1,3}\left(x_{0}, y_{0}, z_{0}\right), \quad f^{2,3}\left(x_{0}, y_{0}, z_{0}\right), \quad f_{3}^{1,2}\left(x_{0}, y_{0}, z_{0}\right)
$$

at any regular point $\left(x_{0}, y_{0}, z_{0}\right)$ of $V$.

Let

$$
\mathcal{S}_{1}=\sum a_{(1)}^{k j} p_{k} p_{j}+W_{(1)}, \quad \mathcal{S}_{2}=\sum a_{(2)}^{k j} p_{k} p_{j}+W_{(2)}
$$

be second-order constants of the motion for a superintegrable system with nondegenerate potential and let $\mathcal{A}_{(i)}(x, y, z)=\left\{a_{(i)}^{k j}(x, y, z)\right\}, i=1,2$ be $3 \times 3$ matrix functions. Then the Poisson bracket of these symmetries is given by

$$
\left\{\mathcal{S}_{1}, \mathcal{S}_{2}\right\}=\sum_{k, j, i=1}^{3} a^{k j i}(x, y, z) p_{k} p_{j} p_{i}+b^{\ell}(x, y, z) p_{\ell},
$$

where

$$
f^{k, \ell}=2 \lambda \sum_{j}\left(a_{(2)}^{k j} a_{(1)}^{j \ell}-a_{(1)}^{k j} a_{(2)}^{j \ell}\right)
$$

Differentiating, we find

$$
f_{i}^{k, \ell}=2 \lambda \sum_{j}\left(\partial_{i} a_{(2)}^{k j} a_{(1)}^{j \ell}+a_{(2)}^{k j} \partial_{i} a_{(1)}^{j \ell}-\partial_{i} a_{(1)}^{k j} a_{(2)}^{j \ell}-a_{(1)}^{k j} \partial_{i} a_{(2)}^{j \ell}\right)+G_{i} f^{k, \ell} .
$$

Clearly, $\left\{\mathcal{S}_{1}, \mathcal{S}_{2}\right\}$ is uniquely determined by the skew-symmetric matrix $\left[\mathcal{A}_{(2)}, \mathcal{A}_{(1)}\right]$ $\equiv \mathcal{A}_{(2)} \mathcal{A}_{(1)}-\mathcal{A}_{(1)} \mathcal{A}_{(2)}$, hence by the constant matrix $\left[\mathcal{A}_{(2)}\left(x_{0}, y_{0}, z_{0}\right), \mathcal{A}_{(1)}\left(x_{0}, y_{0}, z_{0}\right)\right]$ evaluated at a regular point, and by the number $\mathcal{F}\left(x_{0}, y_{0}, z_{0}\right)=f_{3}^{1,2}\left(x_{0}, y_{0}, z_{0}\right)$.

For superintegrable nondegenerate potentials there is a standard structure allowing the identification of the space of second-order constants of the motion with the space $S_{3}$ of $3 \times 3$ symmetric matrices, as well as identification of the space of third-order constants of the motion with a subspace of the space $K_{3} \times F$ of $3 \times 3$ skew-symmetric matrices $K_{3}$, crossed with the line $F$ $=\left\{\mathcal{F}\left(\mathbf{x}_{0}\right)\right\}$. Indeed, if $\mathbf{x}_{0}$ is a regular point then there is a linear correspondence between secondorder symmetries $\mathcal{S}$ and their associated symmetric matrices $\mathcal{A}_{\left(\mathbf{x}_{0}\right)}$. Let $\left\{\mathcal{S}_{1}, \mathcal{S}_{2}\right\}^{\prime}=\left\{\mathcal{S}_{2}, \mathcal{S}_{1}\right\}$ be the reversed Poisson bracket. Then the map 


$$
\left\{\mathcal{S}_{1}, \mathcal{S}_{2}\right\}^{\prime} \Leftrightarrow\left[\mathcal{A}_{(1)}\left(\mathbf{x}_{0}\right), \mathcal{A}_{(2)}\left(\mathbf{x}_{0}\right)\right]
$$

is an algebraic homomorphism. Here, $\mathcal{S}_{1}, \mathcal{S}_{2}$ are in involution if and only if matrices $\mathcal{A}_{(1)}$ $\times\left(\mathbf{x}_{0}\right), \mathcal{A}_{(2)}\left(\mathbf{x}_{0}\right)$ commute and $\mathcal{F}\left(\mathbf{x}_{0}\right)=0$. If $\left\{\mathcal{S}_{1}, \mathcal{S}_{2}\right\} \neq 0$ then it is a third-order symmetry and can be uniquely associated with the skew-symmetric matrix $\left[\mathcal{A}_{(1)}\left(\mathbf{x}_{0}\right), \mathcal{A}_{(2)}\left(\mathbf{x}_{0}\right)\right]$ and the parameter $\mathcal{F}\left(\mathbf{x}_{0}\right)$. Let $\mathcal{E}^{i j}$ be the $3 \times 3$ matrix with a 1 in row $i$, column $j$ and 0 for every other matrix element. Then the symmetric matrices

$$
\mathcal{A}^{(i j)}=\frac{1}{2}\left(\mathcal{E}^{i j}+\mathcal{E}^{j i}\right)=\mathcal{A}^{(j i)}, \quad i, j=1,2,3
$$

form a basis for the 6-dimensional space of symmetric matrices. Moreover,

$$
\left[\mathcal{A}^{(i j)}, \mathcal{A}^{(k \ell)}\right]=\frac{1}{2}\left(\delta_{j k} \mathcal{B}^{(i \ell)}+\delta_{j \ell} \mathcal{B}^{(i k)}+\delta_{i k} \mathcal{B}^{(j \ell)}+\delta_{i \ell} \mathcal{B}^{(j k)}\right),
$$

where

$$
\mathcal{B}^{(i j)}=\frac{1}{2}\left(\mathcal{E}^{i j}-\mathcal{E}^{j i}\right)=-\mathcal{B}^{(j i)}, \quad i, j=1,2,3 .
$$

Here $\mathcal{B}^{(i i)}=0$ and $\mathcal{B}^{(12)}, \mathcal{B}^{(23)}, \mathcal{B}^{(31)}$ form a basis for the space of skew-symmetric matrices. To obtain the commutation relations for the second-order symmetries we need to use relations (42) to compute the parameter $\mathcal{F}\left(\mathbf{x}_{0}\right)$ associated with each commutator $\left[\mathcal{A}^{(i j)}, \mathcal{A}^{(k \ell)}\right]$. The results are straightforward to compute, using relations (27).

$$
\begin{aligned}
& \text { Commutator } \\
& {\left[\mathcal{A}^{(12)}, \mathcal{A}^{(11)}\right]=\mathcal{B}^{(21)}} \\
& {\left[\mathcal{A}^{(13)}, \mathcal{A}^{(11)}\right]=\mathcal{B}^{(31)}} \\
& {\left[\mathcal{A}^{(22)}, \mathcal{A}^{(11)}\right]=0} \\
& {\left[\mathcal{A}^{(23)}, \mathcal{A}^{(11)}\right]=0} \\
& {\left[\mathcal{A}^{(33)}, \mathcal{A}^{(11)}\right]=0} \\
& {\left[\mathcal{A}^{(13)}, \mathcal{A}^{(12)}\right]=\frac{1}{2} \mathcal{B}^{(32)}} \\
& {\left[\mathcal{A}^{(22)}, \mathcal{A}^{(12)}\right]=\mathcal{B}^{(21)}} \\
& {\left[\mathcal{A}^{(23)}, \mathcal{A}^{(12)}\right]=\frac{1}{2} \mathcal{B}^{(31)}} \\
& {\left[\mathcal{A}^{(33)}, \mathcal{A}^{(12)}\right]=0} \\
& {\left[\mathcal{A}^{(22)}, \mathcal{A}^{(13)}\right]=0} \\
& {\left[\mathcal{A}^{(23)}, \mathcal{A}^{(13)}\right]=\frac{1}{2} \mathcal{B}^{(21)}} \\
& {\left[\mathcal{A}^{(33)}, \mathcal{A}^{(13)}\right]=\mathcal{B}^{(31)}} \\
& {\left[\mathcal{A}^{(23)}, \mathcal{A}^{(22)}\right]=\mathcal{B}^{(32)}} \\
& {\left[\mathcal{A}^{(33)}, \mathcal{A}^{(22)}\right]=0} \\
& {\left[\mathcal{A}^{(33)}, \mathcal{A}^{(23)}\right]=\mathcal{B}^{(32)}}
\end{aligned}
$$

$$
\begin{aligned}
& 3 \mathcal{F} / \lambda \\
& -3 A^{13}-B^{23}-G_{3} \\
& A^{12}-B^{33}+G_{2} \\
& -4 A^{23} \\
& 2\left(A^{22}-A^{33}\right) \\
& 4 A^{23} \\
& \frac{1}{2}\left(3 B^{12}-A^{22}+3 A^{33}-G_{1}\right) \\
& -3 B^{23}-A^{13}-G_{3} \\
& \frac{1}{2}\left(-3 B^{33}-3 A^{12}+2 B^{22}+G_{2}\right) \\
& 2\left(B^{23}-A^{13}\right) \\
& -2 B^{33} \\
& -C^{33}+\frac{1}{2} B^{23}-\frac{1}{2} A^{13}-\frac{1}{2} G_{3} \\
& A^{12}+B^{33}+G_{2} \\
& A^{33}-A^{22}-B^{12}-G_{1} \\
& -4 A^{23} \\
& A^{22}-A^{33}-B^{12}-G_{1}
\end{aligned}
$$

Suppose the dimension of the space of truly third-order symmetries generated by commutators of second-order symmetries is 3 . This means that whenever the matrices of two second-order symmetries commute at a regular point $\mathbf{x}_{0}$, so that $f^{1,2}\left(x_{0}, y_{0}, z_{0}\right)=f^{1,3}\left(x_{0}, y_{0}, z_{0}\right)=f^{2,3}\left(x_{0}, y_{0}, z_{0}\right)$ $=0$, then $f_{3}^{1,2}\left(x_{0}, y_{0}, z_{0}\right)=0$. From the table above we see that

$$
A^{23}=0, \quad A^{22}=A^{33}, \quad B^{23}=A^{13}, \quad B^{33}=0 .
$$

Further, since $\left[\mathcal{A}^{(12)}, \mathcal{A}^{(11)}\right]-\left[\mathcal{A}^{(22)}, \mathcal{A}^{(12)}\right]=0$, etc., we have

$$
B^{12}=-\frac{1}{2} A^{33}, \quad B^{22}=2 A^{12}, \quad C^{33}=2 A^{13} .
$$

Substituting these results into the integrability conditions for the potential and the symmetries, we find that there exists a function $U(x, y, z)$ such that

$$
A^{33}=2(\ln U)_{x}, \quad A^{12}=-(\ln U)_{y}, \quad A^{13}=-(\ln U)_{z},
$$

where 


$$
U_{x x}=U_{y y}=U_{z z}, \quad U_{x y}=U_{y z}=U_{z x}=0 .
$$

Note that $U$ is an instance of the isotropic oscillator potential

$$
U=\alpha\left(x^{2}+y^{2}+z^{2}\right)+\beta x+\gamma y+\delta z .
$$

(Further, the defining second-order symmetries for the isotropic oscillator are only weakly functionally independent.) By analogy with the 2D Stäckel transform studied in Ref. 2 (whose 3D form will be studied in our next presentation) it is straightforward to see that the potential of our system is a Stäckel transform by $U$ of the isotropic oscillator potential. By taking the inverse Stäckel transform we can obtain $A^{i j}=B^{i j}=C^{i j} \equiv 0$ for all $i, j$. Plugging these values into the integrability conditions for the symmetries, we find that $G_{1}=G_{2}=G_{3}=0$ so $\lambda$ is a constant. Thus the Stäckel transformed system is just the isotropic harmonic oscillator in flat space.

Corollary 5: Let $V$ be a superintegrable nondegenerate potential on a conformally flat space, not a Stäckel transform of the isotropic oscillator. Then the space of truly third-order constants of the motion is four-dimensional and is spanned by Poisson brackets of the second-order constants of the motion.

\section{THE STANDARD BASIS}

To gain a deeper understanding of our standard basis structure, it is useful to reformulate the problem of determining the second-order symmetries for a nondegenerate superintegrable potential. We set

$$
W(x)=f^{1} V_{1}+f^{2} V_{2}+f^{3} V_{3}+f^{11} V_{11}
$$

and substitute this result into $W_{i}=\lambda \sum_{j=1}^{3} a^{i j} V_{j}$. Additionally we must impose the Killing tensor conditions,

$$
\begin{gathered}
a_{i}^{i i}=-G_{1} a^{1 i}-G_{2} a^{2 i}-G_{3} a^{3 i}, \\
2 a_{i}^{i j}+a_{j}^{i i}=-G_{1} a^{1 j}-G_{2} a^{2 j}-G_{3} a^{3 j}, \quad i \neq j, \\
a_{k}^{i j}+a_{j}^{k i}+a_{i}^{j k}=0, \quad i, j, k \text { distinct. }
\end{gathered}
$$

From the expressions for $W_{i}$ we obtain the equations for the $a^{i j}$,

$$
\begin{gathered}
\lambda a^{11}=f_{1}^{1}+f^{2} A^{12}+f^{3} A^{13}+f^{11} A^{14}, \\
\lambda a^{12}=f_{2}^{1}+f^{1} A^{12}+f^{2} A^{22}+f^{3} A^{32}+f^{11} A^{24}, \\
\lambda a^{13}=f_{3}^{1}+f^{1} A^{13}+f^{2} A^{23}+f^{3} A^{33}+f^{11} A^{34}, \\
\lambda a^{22}=f_{2}^{2}+f^{1} B^{12}+f^{2} B^{22}+f^{3} B^{32}+f^{11} B^{24}, \\
\lambda a^{23}=f_{3}^{2}+f^{1} B^{13}+f^{2} B^{23}+f^{3} B^{33}+f^{11} B^{34}, \\
\lambda a^{33}=f_{3}^{3}+f^{1} C^{13}+f^{2} C^{23}+f^{3} C^{33}+f^{11} C^{34},
\end{gathered}
$$

and the condition on the first derivatives of the $f^{i}$,

$$
\begin{aligned}
& f_{2}^{1}-f_{1}^{2}=-f^{1} A^{12}+f^{2}\left(B^{12}-A^{22}\right)+f^{3}\left(B^{13}-A^{23}\right)+f^{11}\left(B^{14}-A^{24}\right), \\
& f_{3}^{1}-f_{1}^{3}=-f^{1} A^{13}+f^{2}\left(C^{12}-A^{23}\right)+f^{3}\left(C^{13}-A^{33}\right)+f^{11}\left(C^{14}-A^{34}\right),
\end{aligned}
$$




$$
f_{3}^{2}-f_{2}^{3}=f^{1}\left(C^{12}-B^{13}\right)+f^{2}\left(C^{22}-B^{23}\right)+f^{3}\left(C^{23}-B^{33}\right)+f^{11}\left(C^{24}-B^{34}\right) .
$$

Note the expressions for $f_{1}^{11}$ and $f_{2}^{11}$ in terms of $f^{1}, f^{2}, f^{11}$,

$$
f_{1}^{11}=-f^{1}-f^{11}\left(B^{12}-A^{22}\right), \quad f_{2}^{11}=-f^{2}-f^{11} A^{12}, \quad f_{3}^{11}=-f^{3}-f^{11} A^{13} .
$$

Differentiating (46) with respect to each of $x_{1}, x_{2}, x_{3}$ and substituting (45) into the Killing equations we see that we can express each of the second derivatives of $f^{1}, f^{2}, f^{3}$ in terms of lower order derivatives of $f^{1}, f^{2} f^{3}, f^{11}$. Thus the system is in involution at the second derivative level, but not at the first derivative level because we have only three conditions for the nine derivatives $f_{j}^{i}$. We can uniquely determine a symmetry at a regular point by choosing the 10 parameters

$$
\left(f^{1}, f^{2}, f^{3}, f^{11}, f_{1}^{1}, f_{2}^{1}, f_{3}^{1}, f_{2}^{2}, f_{3}^{2}, f_{3}^{3}\right) .
$$

The values of $f^{1}, f^{2}, f^{3}, f^{11}$ at the regular point are analogous to the four parameters that we can add to the potentials in the four parameter family. For our standard basis, we fix $\left(f^{1}, f^{2}, f^{3}, f^{11}\right)_{\mathbf{x}_{0}}=(0,0,0,0)$. Then from (45) and (46) we have

$$
\left(\begin{array}{lll}
f_{1}^{1} & f_{2}^{1} & f_{3}^{1} \\
f_{1}^{2} & f_{2}^{2} & f_{3}^{2} \\
f_{1}^{3} & f_{2}^{3} & f_{3}^{3}
\end{array}\right)=\lambda\left(\begin{array}{ccc}
a^{11} & a^{12} & a^{13} \\
a^{21} & a^{22} & a^{23} \\
a^{31} & a^{32} & a^{33}
\end{array}\right) .
$$

Thus we can define a standard set of basis symmetries $\mathcal{S}^{(j k)}=\sum a^{i j}(\mathbf{x}) p_{i} p_{j}+W^{(i j)}(\mathbf{x})$ corresponding to a regular point $\mathbf{x}_{0}$ by

$$
\frac{1}{\lambda}\left(\begin{array}{lll}
f_{1}^{1} & f_{2}^{1} & f_{3}^{1} \\
f_{1}^{2} & f_{2}^{2} & f_{3}^{2} \\
f_{1}^{3} & f_{2}^{3} & f_{3}^{3}
\end{array}\right)_{\mathbf{x}_{0}}=\left(\begin{array}{ccc}
a^{11} & a^{12} & a^{13} \\
a^{21} & a^{22} & a^{23} \\
a^{31} & a^{32} & a^{33}
\end{array}\right)_{\mathbf{x}_{0}}=\mathcal{A}^{(j k)}, \quad W^{(j k)}\left(\mathbf{x}_{0}\right)=0 .
$$

The condition on $W^{(j k)}$ is actually four conditions since $W^{(j k)}$ depends on four parameters.

\section{MAXIMUM DIMENSIONS OF THE SPACES OF POLYNOMIAL CONSTANTS}

In order to demonstrate the existence and structure of quadratic algebras for 3D superintegrable systems on conformally flat spaces, it is important to compute the dimensions of the spaces of symmetries of these systems that are of orders 4 and 6 . These symmetries are necessarily of a special type. The highest order terms in the momenta are independent of the parameters in the potential, while the terms of order 2 less in the momenta are linear in these parameters, those of order 4 less are quadratic, and those of order 6 less are cubic. We will obtain these dimensions exactly, but first we need to establish sharp upper bounds.

\section{A. Quartic constants}

We investigate the space of fourth-order constants of the motion

$$
\mathcal{F}=\sum_{\ell, k, j, i=1}^{3} a^{\ell k j i}(x, y, z) p_{\ell} p_{k} p_{j} p_{i}+\sum_{m, q=1}^{3} b^{m q}(x, y, z) p_{m} p_{q}+W(x, y, z),
$$

which must satisfy $\{\mathcal{H}, \mathcal{F}\}=0$. Here $a^{\ell k j i}, b^{m q}$ are symmetric in all indices.

The conditions are

$$
a_{i}^{i i i i}=-2 \sum_{s} \frac{\lambda_{s}}{\lambda} a^{s i i i}
$$




$$
\begin{gathered}
4 a_{i}^{j i i i}+a_{j}^{i i i i}=-6 \sum_{s} \frac{\lambda_{s}}{\lambda} a^{s j i i}, \quad i \neq j, \\
3 a_{i}^{j j i i}+2 a_{j}^{i i i j}=-\sum_{s} \frac{\lambda_{s}}{\lambda} a^{s i i i}-3 \sum_{s} \frac{\lambda_{s}}{\lambda} a^{s i j j}, \quad i \neq j, \\
3 a_{i}^{i i j k}+a_{j}^{k i i i}+a_{k}^{j i i i}=-6 \sum_{s} \frac{\lambda_{s}}{\lambda} a^{s i j k}, \quad i, j, k \text { distinct, } \\
2 a_{i}^{i j j k}+2 a_{j}^{j i i k}+a_{k}^{i i j j}=-\sum_{s} \frac{\lambda_{s}}{\lambda}\left(a^{s k i i}+a^{s k j j}\right), \quad i, j, k \text { distinct }, \\
b_{i}^{j k}+b_{k}^{i j}+b_{j}^{k i}=6 \lambda \sum_{s} a^{s k j i} V_{s}, \quad i, j, k \text { distinct }, \\
b_{i}^{i i}=2 \lambda \sum_{s=1} a^{s i i i} V_{s}-\sum_{s} \frac{\lambda_{s}}{\lambda} b^{s i}, \\
b_{i}^{i j}+b_{j}^{i i}=6 \lambda \sum_{s} a^{s j i i} V_{s}-\sum_{s} \frac{\lambda_{s}}{\lambda} b^{s i}, \quad i \neq j,
\end{gathered}
$$

and

$$
\lambda \sum_{s} b^{s i} V_{s}=W_{i}
$$

Clearly, the $a^{\ell k j i}$ is a fourth-order Killing tensor. We require the potential $V$ to be superintegrable and nondegenerate. Also we require that the highest order terms, the $a^{\ell k j i}$ in the constant of the motion be independent of the four independent parameters in $V$. However, the $b^{m q}$ must depend linearly and $W$ quadratically on these parameters.

We set

$$
b^{j k}=\sum_{\alpha=1}^{4} f^{j k, \alpha} W^{(\alpha)}, \quad f^{j k, \alpha}=f^{k j, \alpha},
$$

where $W^{(\alpha)}$ is defined by (18). Then conditions (49) take the form

$$
\partial_{x_{h}} f^{j k, \alpha}+\partial_{x_{k}} f^{h j, \alpha}+\partial_{x_{j}} f^{k h, \alpha}-\lambda a^{\alpha h j k}=\cdots,
$$

where the right-hand side depends only on the $f^{j k, \alpha}, 1 \leqslant j, k, h \leqslant 3$ and we set $a^{4 h j k} \equiv 0$. From the integrability conditions $\partial_{x_{j}}\left(\partial W / \partial x_{i}\right)=\partial_{x_{i}}\left(\partial W / \partial x_{j}\right), i \neq j$ for Eq. (50) we obtain the conditions

$$
\partial_{x_{j}} f^{\beta k, \alpha}+\partial_{x_{j}} f^{\alpha k, \beta}-\partial_{x_{k}} f^{\beta j, \alpha}-\partial_{x_{k}} f^{\alpha j, \beta}=\cdots,
$$

where the right-hand side depends only on the $f^{j k, \alpha}, j \neq k, 1 \leqslant \alpha, \beta \leqslant 4$ and we set $f^{4 j, \alpha} \equiv 0$.

There are 30 independent equations (51) with $\alpha \neq 4$ and we use 15 of these to define the 15 components $a^{i h j k}$ as linear combinations of $\partial_{x_{h}} f^{j k, \alpha}$ and $f^{j k, \alpha}$. We can then eliminate the $a^{i h j k}$ from the remaining 15 equations to obtain 15 conditions relating $\partial_{x_{h}} f^{j k, \alpha}$ and $f^{j k, \alpha}$. There are 18 terms of the form $\partial_{x_{h}} f^{j k, 4}$. Equations (52) with $\alpha=\beta=4$ are satisfied identically. There are nine equations (52) with $\beta=4,1 \leqslant \alpha \leqslant 3$ and 10 equations (51) with $\alpha=4$. Thus all terms of the form $\partial_{x_{h}} f^{j k, 4}$ can be expressed as linear combinations of $f^{j k, \alpha}$. There are a total of 54 distinct terms of the form 
$\partial_{x_{h}} f^{j k, m}, 1 \leqslant h, j, k, m \leqslant 3$. We have seen that there are 15 conditions on these terms remaining from (51); there are an additional 18 such conditions from (52) with $\alpha, \beta \neq 4$. Thus there is a shortfall of 21 conditions on the first derivatives $\partial_{x_{h}} f^{j k, m}$.

There are a total of 108 distinct terms of the form $\partial_{x_{h}} \partial_{x_{\ell}} f^{j k, m}$ with $1 \leqslant h j, k, \ell, m \leqslant 3$. Differentiating with respect to $x_{1}, x_{2}$, and $x_{3}$ the 15 first-order conditions of (51), from which the $a^{i h j k}$ have been eliminated, we obtain 45 independent conditions on these second derivatives. Differentiating each of our expressions for the $a^{i h j k}$ and substituting into equations (48) we find 63 additional conditions on the second derivatives. This allows us to express each second-order derivative as a linear combination of lower order derivatives, Thus the system is in involution. Also, we can differentiate the 18 equations from (52) with $\alpha, \beta \neq 4$ to obtain 54 additional conditions on the second derivatives (which may or may not be independent of those already found).

We conclude that any fourth-order symmetry is uniquely determined by the values $f^{j k, \alpha}\left(\mathbf{x}_{0}\right)$ and a subset of 21 of the values $\partial_{x_{h}}{ }^{j k, m}\left(\mathbf{x}_{0}\right)$ at a regular point $\mathbf{x}_{0}$. Note that by adding an appropriate linear combination of purely second-order symmetries to the fourth-order symmetry we can achieve $f^{j k, \alpha}\left(\mathbf{x}_{0}\right)=0$ for all $j, k, \alpha$, so the maximum possible dimension of the space of purely fourth-order symmetries for a nondegenerate potential is 21 .

\section{B. Sixth-order constants}

We take the general sixth-order symmetry for a nondegenerate potential to be of the form

$$
\mathcal{L}=\sum a^{h i j k l t} p_{h} p_{i} p_{j} p_{k} p_{l} p_{t}+\sum b^{h i j k} p_{h} p_{i} p_{j} p_{k}+\sum c^{i j} p_{i} p_{j}+W,
$$

where the functions $a^{h i j k l t}, b^{h i j k}, c^{i j}$ are symmetric in all indices. Here $a^{h i j k l t}$ is independent of the parameters $V_{\alpha}, \alpha=1, \ldots, 4, b^{h i j k}$ is a homogeneous quadratic polynomial in the $W^{(\alpha)}, c^{i j}$ is homogeneous fourth order, and $W$ is homogeneous sixth order in the $W^{(\alpha)}$. The Poisson bracket of $\mathcal{H}$ and $\mathcal{L}$ is polynomial in the momenta and the parameters $W^{(\alpha)}$ and for it to vanish at a regular point, each coefficient of this polynomial must vanish separately. The conditions are (for $1 \leqslant i, j, k \leqslant k$ and $i, j, k$ pairwise distinct and for $s=1,2,3$ )

$$
\begin{gathered}
a_{i}^{i i i i i i}=-3 \sum_{s} \frac{\lambda_{s}}{\lambda} a^{s i i i i i}, \\
6 a_{i}^{j i i i i i}+a_{j}^{i i i i i i}=-15 \sum_{s} \frac{\lambda_{s}}{\lambda} a^{s j i i i i}, \\
5 a_{i}^{j j i i i i}+2 a_{j}^{j i i i i i}=-\sum_{s} \frac{\lambda_{s}}{\lambda} a^{s i i i i i}-10 \sum_{s} \frac{\lambda_{s}}{\lambda} a^{s j j i i i}, \\
4 a_{i}^{j j j i i i}+3 a_{j}^{j j i i i i}=-3 \sum_{s} \frac{\lambda_{s}}{\lambda} a^{s j i i i i}-12 \sum_{s} \frac{\lambda_{s}}{\lambda} a^{s j j j i i}, \\
5 a_{i}^{j k i i i i}+a_{k}^{j i i i i i}+a_{j}^{k i i i i i}=-10 \sum_{s} \frac{\lambda_{s}}{\lambda} a^{s j k i i i}, \\
4 a_{i}^{j j k i i i}+2 a_{j}^{j k i i i i}+a_{k}^{j j i i i i}=-6 \sum_{s} \frac{\lambda_{s}}{\lambda} a^{s j j k i i}-\sum_{s} \frac{\lambda_{s}}{\lambda} a^{s k i i i i},
\end{gathered}
$$




$$
\begin{gathered}
3 a_{i}^{j j k k i i}+2 a_{j}^{j k k i i i}+2 a_{k}^{k j j i i i}=-90 \sum_{s} \frac{\lambda_{s}}{\lambda} a^{s i j j k k}-30 \sum_{s} \frac{\lambda_{s}}{\lambda} a^{s k k i i i}-30 \sum_{s} \frac{\lambda_{s}}{\lambda} a^{s j j i i i}, \\
b_{i}^{i i i i}=3 \lambda \sum_{s} a^{s i i i i i} W^{(s)}-2 \sum_{s} \frac{\lambda_{s}}{\lambda} b^{s i i i}, \\
b_{j}^{i i i i}+4 b_{i}^{j i i i}=15 \lambda \sum_{s} a^{s j i i i i} W^{(s)}-6 \sum_{s} \frac{\lambda_{s}}{\lambda} b^{s j i i}, \\
2 b_{j}^{i i i j}+3 b_{i}^{j j i i}=15 \lambda \sum_{s} a^{s j j i i i} W^{(s)}-\sum_{s} \frac{\lambda_{s}}{\lambda}\left(2 b^{s i i i}+6 b^{s j j i}\right), \\
\sum_{j}^{i i i k}+b_{k}^{i i i j}+3 b_{i}^{i i j k}=15 \lambda \sum_{s}^{s i} a^{s i i i j k} W^{(s)}-6 \sum_{s} \frac{\lambda_{s}}{\lambda} b^{s i j k}, \\
c_{i}^{i i j}=2 \lambda \sum_{s} b^{s i i i} W^{(s)}-\sum_{s}^{i j} \frac{\lambda_{s}}{\lambda} c^{s i}, \\
b_{k}^{i i j j}+2 b_{j}^{i i j k}+2 b_{i}^{i j j k}=15 \lambda \sum_{s} a^{s i i j}=6 \lambda \sum_{s} \sum_{s}^{s i j k} W^{(s)}-2 \sum_{s} \frac{\lambda_{s}}{\lambda}\left(b^{s i i j k}+b^{s j j k}\right),
\end{gathered}
$$

We set

$$
c^{i j}=\sum_{\alpha, \beta=1}^{4} c^{i j, \alpha \beta} W^{(\alpha)} W^{(\beta)}, \quad c^{i j, \alpha \beta}=c^{i j, \beta \alpha} .
$$

There are $6 \times 10=60$ independent terms $c^{i j, \alpha \beta}$. There are $60 \times 3=180$ terms $c_{k}^{i j, \alpha \beta}$ of which 108 are of the form $c_{k}^{i j, s t}, 54$ are of the form $c_{k}^{i j, 4 s}$, and 18 are of the form $c_{k}^{i j, 44}$. Equations (56) give 30 conditions relating the derivatives $c_{k}^{i j, \alpha \beta}, 18$ conditions relating the derivatives $c_{k}^{i j, 4 s}$, and 8 conditions relating the derivatives $c_{k}^{i j, 44}$. The 100 independent equations (55) allow us to solve for the 15 terms $b^{i j k \ell, 4}$ and the 45 terms $b^{i j k \ell, s}$ where

$$
b^{i j k \ell}=\sum_{\alpha=1}^{4} b^{i j k \ell, \alpha} W^{(\alpha)} .
$$

Further, they yield 10 conditions relating the derivatives $c_{k}^{i j, 44}, 15$ equations relating the derivatives $c_{k}^{i j, 4 s}$, and 15 equations relating the derivatives $c_{k}^{i j, s t}$. It follows that all 18 terms of the form $c_{k}^{i j, 44}$ 
can be expressed as linear combinations of the $c^{i j, \alpha \beta}$. There are a total of 78 conditions on the remaining 162 terms.

There are 360 terms $c_{k \ell}^{i j, \alpha \beta}$ of which we can ignore the 36 terms $c_{k \ell}^{i j, 44}$. The 84 equations (54) allow us to solve for the 28 terms $a^{h i j k \ell m}$ and give 21 conditions for $c_{k \ell}^{i j, 4 s}$ and 35 conditions for $c_{k \ell}^{i j, s t}$. Further differentiating our previously obtained 78 conditions on the first derivatives we obtain $78 \times 3=234$ conditions, 99 on $c_{k \ell}^{i j, s t}$ and 135 independent conditions on $c_{k \ell}^{i j, s t}$. It follows that all 108 terms of the form $c_{k \ell}^{i j, 4 s}$ can be expressed as linear combinations of lower order terms and there is a total of $35+135=160$ independent conditions on the 216 terms $c_{k \ell}^{i j, s t}$. Finally, differentiating the previous conditions obtained for the $c_{k \ell}^{i j, s t}$ and using the 31 equations (53) we obtain at least 360 independent conditions for the 360 terms $c_{\mathrm{k \ell m}}^{i j, s t}$. Thus the maximal number of parameters in a solution of the sixth order symmetry equations is $60+84+56=200$, excluding the 35 independent additive terms $W^{(\alpha)} W^{(\beta)} W^{(\gamma)}$.

We know that the dimension of the space of second-order symmetries for a superintegrable system with nondegenerate potential is 6 . Now let us suppose (as we will prove) the dimension of the space of quartic symmetries is 21 . Then there are exactly $84=21 \times 4$ independent sixth-order symmetries that are also quartic symmetries, and $6 \times 10=60$ independent sixth-order symmetries that are also quadratic. Thus the maximal possible dimension of the space of truly sixth-order symmetries is $200-84-60=56$. We will show that this bound of 56 is actually achieved.

\section{BASES FOR THE FOURTH- AND SIXTH-ORDER CONSTANTS OF THE MOTION}

It follows from Sec. VII A that, for a superintegrable system with nondegenerate potential, the dimension of the space of truly fourth-order constants of the motion is at most 21. Note from Sec. VI that at any regular point $\mathbf{x}_{0}$, we can define a standard basis of six second-order constants of the motion $\mathcal{S}^{(i j)}=A^{(i j)}+W^{(i j)}$ where the quadratic form $A^{(i j)}$ has matrix $\mathcal{A}^{(i j)}$ defined by (43) and $W^{(i j)}$ is the potential term with $W^{(i j)}\left(\mathbf{x}_{0}\right) \equiv 0$ identically in the parameters $W^{(\alpha)}$. By taking homogeneous polynomials of order two in the standard basis symmetries we can construct fourth order symmetries.

Question: Is every fourth-order symmetry a polynomial in the second-order symmetries?

Answer: Yes Also the dimension of the space of fourth-order symmetries is exactly 21.

Theorem 5: The 21 distinct standard monomials $\mathcal{S}^{(i j)} \mathcal{S}^{(j k)}$, defined with respect to a regular point $\mathbf{x}_{0}$, form a basis for the space of fourth-order symmetries.

Proof: We choose the basis symmetries in the form

$$
\begin{gathered}
(1)\left(\mathcal{S}^{(i i)}\right)^{2}, \mathcal{S}^{(i i)} \mathcal{S}^{(i j)}, \mathcal{S}^{(i i)} \mathcal{S}^{(j j)}, \mathcal{S}^{(i i)} \mathcal{S}^{(j k)} \\
\text { (2) } \mathcal{S}^{(i i)} \mathcal{S}^{(j j)}-\left(\mathcal{S}^{(i j)}\right)^{2} \\
\text { (3) } \mathcal{S}^{(i j)} \mathcal{S}^{(i k)}-\mathcal{S}^{(i i)} \mathcal{S}^{(j k)}
\end{gathered}
$$

for $i, j, k=1, \ldots, 3 i, j, k$ pairwise distinct (three possibilities).

If we evaluate this set at the regular point the first class of symmetries will be $p_{i}^{4}, p_{i}^{3} p_{j} p_{i}^{2} p_{j}^{2}, p_{i}^{2} p_{j} p_{k}$, respectively, whereas the last two classes of symmetries will vanish. Thus the only possible linear dependencies are those relating the six symmetries

$$
\begin{gathered}
F^{(12)}=\mathcal{S}^{(11)} \mathcal{S}^{(22)}-\left(\mathcal{S}^{(12)}\right)^{2}, \quad F^{(13)}=\mathcal{S}^{(11)} \mathcal{S}^{(33)}-\left(\mathcal{S}^{(13)}\right)^{2}, \quad F^{(23)}=\mathcal{S}^{(22)} \mathcal{S}^{(33)}-\left(\mathcal{S}^{(23)}\right)^{2}, \\
G^{(23)}=\mathcal{S}^{(12)} \mathcal{S}^{(13)}-\mathcal{S}^{(11)} \mathcal{S}^{(23)}, \quad G^{(13)}=\mathcal{S}^{(12)} \mathcal{S}^{(23)}-\mathcal{S}^{(22)} \mathcal{S}^{(13)}, \quad G^{(12)}=\mathcal{S}^{(13)} \mathcal{S}^{(23)}-\mathcal{S}^{(33)} \mathcal{S}^{(12)}
\end{gathered}
$$

The second-order terms in the symmetry $F^{(i j)}$ are

$$
\mathcal{F}^{(i j)}=\mathcal{A}^{(i i)} W^{(j j)}+\mathcal{A}^{(j j)} W^{(i i)}-2 \mathcal{A}^{(i j)} W^{(i j)} .
$$

Now $\mathcal{F}^{(i j)}$ vanishes at the regular point but its derivatives at the point are 


$$
\mathcal{F}_{i}^{(i j)}=p_{j}^{2} V_{i}-p_{i} p_{j} V_{j}, \quad \mathcal{F}_{j}^{(i j)}=p_{i}^{2} V_{j}-p_{j} p_{i} V_{i}, \quad \mathcal{F}_{k}^{(i j)}=0 .
$$

Similarly the second-order terms in the symmetry $G^{(j k)}$ are

$$
\mathcal{G}^{(j k)}=\mathcal{A}^{(i j)} W^{(i k)}+\mathcal{A}^{(i k)} W^{(i j)}-\mathcal{A}^{(i i)} W^{(j k)}-\mathcal{A}^{(j k)} W^{(i i)} .
$$

Again $\mathcal{G}^{(i j)}$ vanishes at the regular point but its derivatives at the point are

$$
\mathcal{G}_{i}^{(j k)}=\frac{1}{2} p_{i} p_{j} V_{k}+\frac{1}{2} p_{i} p_{k} V_{j}-p_{j} p_{k} V_{i}, \quad \mathcal{G}_{j}^{(j k)}=\frac{1}{2} p_{i} p_{k} V_{i}-\frac{1}{2} p_{i}^{2} V_{k}, \quad \mathcal{G}_{k}^{(j k)}=\frac{1}{2} p_{i} p_{j} V_{i}-\frac{1}{2} p_{i}^{2} V_{j} .
$$

Since $V_{1}, V_{2}, V_{3}$ are arbitrary, it is clear that these six terms are linearly independent. Thus the 21 symmetries form a basis.

Q.E.D.

Now we know that for a superintegrable system with nondegenerate potential the space of purely fourth-order constants of the motion is exactly 21. Thus from Sec. VII B the dimension of the space of purely sixth-order constants of the motion is at most 56 . Again we shall show that the 56 independent homogeneous third-order polynomials in the symmetries $\mathcal{S}^{(i j)}$ form a basis for this space.

At the sixth-order level we have the symmetries

$$
\text { (1) }\left(\mathcal{S}^{(i i)}\right)^{3},\left(\mathcal{S}^{(i i)}\right)^{2} \mathcal{S}^{(i j)},\left(\mathcal{S}^{(i i)}\right)^{2} \mathcal{S}^{(j j)},\left(\mathcal{S}^{(i i)}\right)^{2} \mathcal{S}^{(j k)}
$$

for $i, j, k=1, \ldots, 3 i, j, k$ pairwise distinct (18 possibilities),

$$
\text { (2) } \mathcal{S}^{(i i)} \mathcal{S}^{(i j)} \mathcal{S}^{(j j)}, \mathcal{S}^{(i i)} \mathcal{S}^{(i j)} \mathcal{S}^{(j k)}, \mathcal{S}^{(i i)} \mathcal{S}^{(j j)} \mathcal{S}^{(k k)}
$$

$$
\text { (3) } \mathcal{S}^{(\ell m)}\left(\mathcal{S}^{(i i)} \mathcal{S}^{(j j)}-\left(\mathcal{S}^{(i j)}\right)^{2}\right)
$$

$$
\text { (4) } \mathcal{S}^{(\ell m)}\left(\mathcal{S}^{(i j)} \mathcal{S}^{(i k)}-\mathcal{S}^{(i i)} \mathcal{S}^{(j k)}\right)
$$

for $i, j, k=1, \ldots, 3 i, j, k$ pairwise distinct (18 possibilities).

Theorem 6: The 56 distinct standard monomials $\mathcal{S}^{(h i)} \mathcal{S}^{(j k)} \mathcal{S}^{(\ell m)}$, defined with respect to a regular $\mathbf{x}_{0}$, form a basis for the space of sixth-order symmetries.

Proof: Rather than using the monomials directly we choose the polynomials in the forms (1)-(4) above. Suppose some linear combination $\mathcal{C}$ of these 56 polynomials has identically vanishing sixth-order terms. This implies immediately that the coefficients of the first 28 polynomials are zero. Thus $\mathcal{C}$ must be a linear combination of the six fourth-order symmetries $F^{(i j)}, G^{(i j)}$ of Theorem 5. Now the first derivatives of the second-order terms in $\mathcal{C}$ all vanish at $\mathbf{x}_{0}$ so by the proof of Theorem 5 the linear combination of fourth-order basis symmetries must vanish. Thus we must have $\mathcal{C} \equiv 0$. Then evaluating the expressions

$$
\partial_{x_{h}} \mathcal{C}\left(\mathbf{x}_{0}\right)=0, \quad \partial_{x_{h} x_{\ell}}^{2} \mathcal{C}\left(\mathbf{x}_{0}\right)=0 .
$$

and making use of the expressions for $\mathcal{F}^{i j}, \mathcal{G}^{i j}$ in the proof of Theorem 5 it is straightforward to show that the coefficients of all 28 terms in $\mathcal{C}$ must vanish. The 56 terms are linearly independent and all nonzero linear combinations are truly sixth order.

Q.E.D.

We conclude that the quadratic algebra closes.

${ }^{1}$ E. G. Kalnins, J. M. Kress, and W. Miller, Jr., J. Math. Phys. 46, 053509 (2005).

${ }^{2}$ E. G. Kalnins, J. M. Kress, and W. Miller, Jr., J. Math. Phys. 46, 053510 (2005).

${ }^{3}$ S. Wojciechowski, Phys. Lett. A 95, 279 (1983).

${ }^{4}$ N. W. Evans, Phys. Rev. A 41, 5666 (1990); J. Math. Phys. 32, 3369 (1991).

${ }^{5}$ N. W. Evans, Phys. Lett. A 147, 483 (1990).

${ }^{6}$ J. Friš, V. Mandrosov, Ya. A. Smorodinsky, M. Uhlír, and P. Winternitz, Phys. Lett. 16, 354 (1965).

${ }^{7}$ J. Friš, Ya. A. Smorodinskii, M. Uhlír, and P. Winternitz, Sov. J. Nucl. Phys. 4, 444 (1967).

${ }^{8}$ A. A. Makarov, Ya. A. Smorodinsky, Kh. Valiev, and P. Winternitz, Nuovo Cimento A 52, 1061 (1967).

${ }^{9}$ F. Calogero, J. Math. Phys. 10, 2191 (1969).

${ }^{10}$ A. Cisneros and H. V. McIntosh, J. Math. Phys. 10, 277 (1969).

${ }^{11}$ E. K. Sklyanin, J. Sov. Math. 47, 2473 (1989). 
${ }^{12}$ L. D. Faddeev and L. A. Takhtajan, Hamiltonian Methods in the Theory of Solitons (Springer, Berlin, 1987).

${ }^{13}$ A. G. Ushveridze, Quasi-Exactly Solvable Models in Quantum Mechanics (Institute of Physics, Bristol, 1993).

${ }^{14}$ J. Harnad, "Loop groups, R-matrices and separation of variables," in Integrable systems: from classical to quantum, edited by J. Harnad, G. Sabidussi, and P. Winternitz, CRM Proceedings and Lecture Notes, Vol. 26 (American Mathematical Society, Providence, Rhode Island, 2000), pp. 21-54.

${ }^{15}$ M. Karlovini and K. Rosquist, J. Math. Phys. 41, 370 (2000).

${ }^{16}$ L. P. Eisenhart, Riemannian Geometry (Princeton University Press, Princeton, NJ, 1949).

${ }^{17}$ W. Miller, Jr., Symmetry and Separation of Variables (Addison-Wesley, Providence, RI, 1977).

${ }^{18}$ E. G. Kalnins and W. Miller, Jr., SIAM J. Math. Anal. 11, 1011 (1980).

${ }^{19}$ W. Miller, "The technique of variable separation for partial differential equations," Proceedings of School and Workshop on Nonlinear Phenomena, Oaxtepec, Mexico, November 29-December 17, 1982, Lecture Notes in Physics, Vol. 189 (Springer-Verlag, New York, 1983), pp. 184-208.

${ }^{20}$ E. G. Kalnins, Separation of Variables for Riemannian Spaces of Constant Curvature, Pitman, Monographs and Surveys in Pure and Applied Mathematics 28 (Longman, Essex, England, 1986).

${ }^{21}$ W. Miller, Jr., Mechanisms for variable separation in partial differential equations and their relationship to group theory," Symmetries and Nonlinear Phenomena (World Scientific, Singapore, 1988), pp. 188-221.

${ }^{22}$ E. G. Kalnins, W. Miller, Jr., and G. S. Pogosyan, J. Math. Phys. 40, 708 (1999).

${ }^{23}$ E. G. Kalnins, W. Miller, Jr., and G. S. Pogosyan, J. Math. Phys. 37, 6439 (1996).

${ }^{24}$ D. Bonatos, C. Daskaloyannis, and K. Kokkotas, Phys. Rev. A 50, 3700 (1994).

${ }^{25}$ C. Daskaloyannis, J. Math. Phys. 42, 1100 (2001).

${ }^{26}$ S. P. Smith, Trans. Am. Math. Soc. 322, 285 (1990).

${ }^{27}$ E. G. Kalnins, W. Miller, and M. V. Tratnik, SIAM J. Math. Anal. 22, 272 (1991).

${ }^{28}$ P. Letourneau and L. Vinet, Ann. Phys. (N.Y.) 243, 144 (1995).

${ }^{29}$ C. Grosche, G. S. Pogosyan, and A. N. Sissakian, Fortschr. Phys. 43, 453 (1995).

${ }^{30}$ E. G. Kalnins, J. M. Kress, W. Miller, Jr., and G. S. Pogosyan, J. Phys. A 34, 4705 (2001).

${ }^{31}$ E. G. Kalnins, J. M. Kress, and P. Winternitz, J. Math. Phys. 43, 970 (2002).

${ }^{32}$ E. G. Kalnins, J. M. Kress, W. Miller, Jr., and P. Winternitz, J. Math. Phys. 44, 5811 (2003).

${ }^{33}$ M. F. Rañada, J. Math. Phys. 38, 4165 (1997).

${ }^{34}$ E. G. Kalnins, W. Miller, Jr., G. C. Williams, and G. S. Pogosyan, J. Phys. A 35, 4655 (2002).

${ }^{35}$ C. P. Boyer, E. G. Kalnins, and W. Miller, SIAM J. Math. Anal. 17, 778 (1986).

${ }^{36}$ J. Hietarinta, B. Grammaticos, B. Dorizzi, and A. Ramani, Phys. Rev. Lett. 53, 1707 (1984). 